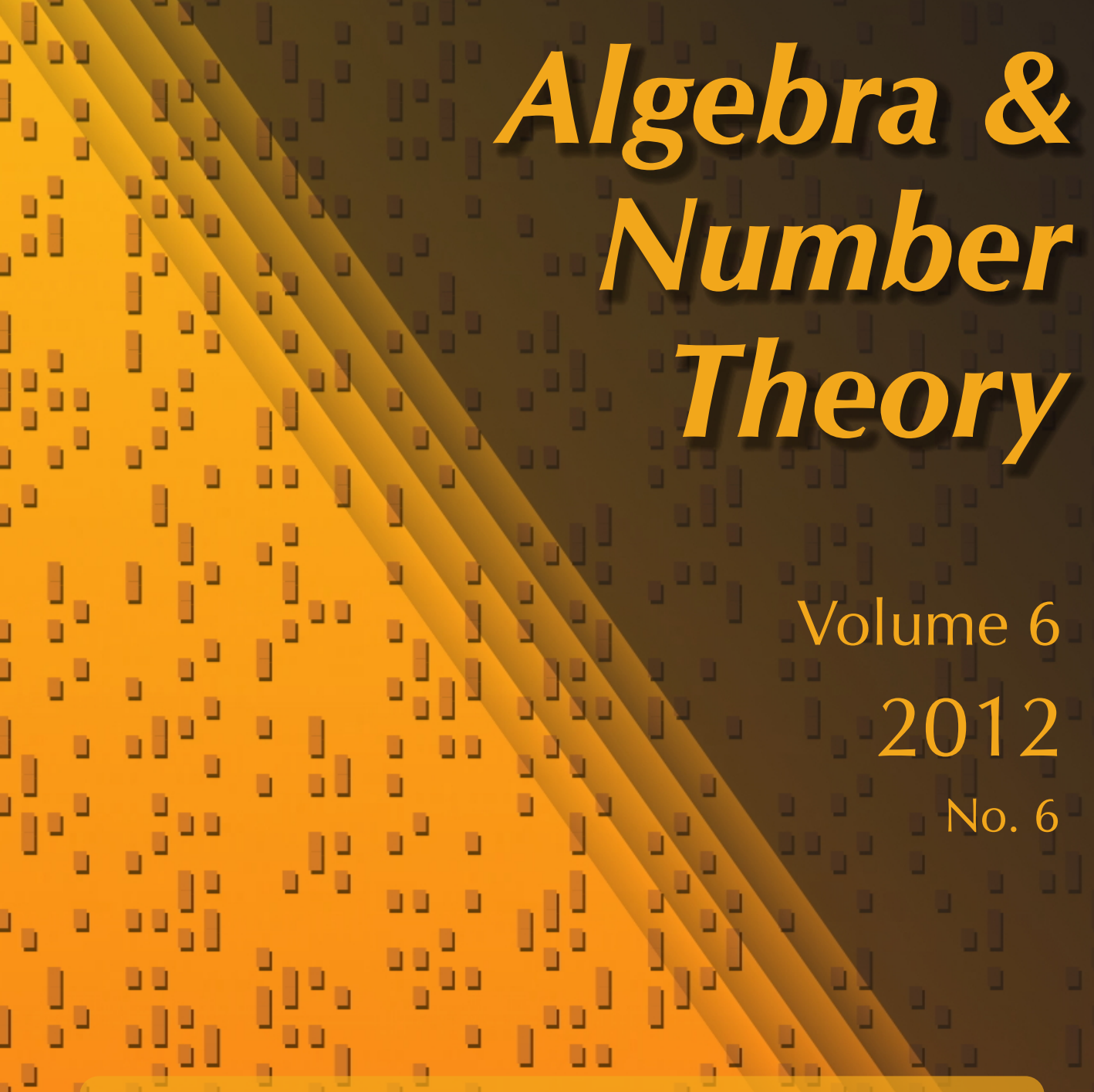

On the geometric realization of the inner product

and canonical basis

for quantum affine $\mathfrak{s l}_{n}$

」

$$
\lrcorner\lrcorner \quad \text { Kevin McGerty }\lrcorner\lrcorner
$$$$
\lrcorner\lrcorner\lrcorner\lrcorner\lrcorner\rfloor
$$$$
\rfloor
$$

$\perp-\lrcorner$ 


\title{
On the geometric realization of the inner product and canonical basis for quantum affine $\mathfrak{s l}_{n}$
}

\author{
Kevin McGerty
}

\begin{abstract}
We give a geometric interpretation of the inner product on the modified quantum group of $\widehat{\mathfrak{s l}}_{n}$. We also give some applications of this interpretation, including a positivity result for the inner product, and a new geometric construction of the canonical basis.
\end{abstract}

\section{Introduction}

Let $\mathbf{U}$ be a quantized universal enveloping algebra. The positive part $\mathbf{U}^{+}$of $\mathbf{U}$ is well known to possess a canonical basis [Lusztig 1990; Kashiwara 1991; Lusztig 1991]. In contrast, there is no natural basis for the algebra $\mathbf{U}$ itself. However Lusztig [1992] has defined a variant of the quantized enveloping algebra known as the modified quantum group. This algebra has essentially the same representation theory, and can be given a canonical basis $\dot{\mathbf{B}}$ which packages together natural bases of the tensor product of a highest and lowest weight $\mathbf{U}$-module (when such modules exist), in the same way that the canonical basis $\mathbf{B}$ of $\mathbf{U}^{+}$packages together natural bases of highest weight representations. Just as for $\mathbf{B}$ (see [Grojnowski and Lusztig 1993; Kashiwara 1991]) it is possible to characterise this basis, up to sign, in terms of an involution and an inner product.

In [Beilinson et al. 1990] the quantized enveloping algebra of $\mathfrak{g l}_{n}$ was constructed geometrically as a limit of certain convolution algebras. Subsequently Lusztig [1993, Part 4, Notes 1; 1999], and independently Ginzburg and Vasserot [1993], observed that this construction could be extended to the case of quantum affine $\mathfrak{s l}_{n}$. More precisely, one can define a sequence of algebra $\mathfrak{A}_{D}$, and maps $\psi_{D}: \mathfrak{A}_{D} \rightarrow \mathfrak{A}_{D-n}$, along with compatible maps $\phi_{D}$ from the modified quantum group $\dot{\mathbf{U}}\left(\widehat{\mathfrak{s l}}_{n}\right)$ such that the resulting map into the inverse limit of the system $\left(\mathfrak{A}_{D}, \psi_{D}\right)_{D \in \mathbb{N}}$ is injective (in fact we will give a new proof of this injectivity statement in Section 7). One of the main results of the present paper is a construction of the inner product on

MSC2010: primary 20G42; secondary 20G43, 17B37.

Keywords: quantum groups, canonical bases, perverse sheaves. 
the modified quantum group $\dot{\mathbf{U}}\left(\widehat{\mathfrak{s l}}_{n}\right)$ as a kind of limit of a natural family of inner products on the algebras $\left(\mathfrak{A}_{D}\right)_{D \in \mathbb{N}}$. We do this both in the context of function on $\mathbb{F}_{q}$-rational points and perverse sheaves, giving two proofs of the fact that our construction yields the inner product on the modified quantum group - the first is elementary, using explicit formulae for multiplication in $\mathfrak{A}_{D, n, n}$, while the second, although requiring more machinery, gives a more conceptual explanation in terms of equivariant cohomology. As applications of these results we give a new construction of the canonical basis of $\dot{\mathbf{U}}$ in this context (which was already shown in [Schiffmann and Vasserot 2000] using crystal basis techniques), and a prove a positivity property for the inner product of elements of $\dot{\mathbf{B}}$ which is conjectured to hold for arbitrary types.

\section{Background}

We begin by recalling the setup of [Lusztig 1999]. Fix a positive integer $n$. Let $D$ be a positive integer, $\epsilon$ an indeterminate, $\mathbb{k}$ a finite field with $q$ elements and $v$ a square root of $q$. Given a free $\mathbb{k}\left[\epsilon, \epsilon^{-1}\right]$-module $V$ of rank $D$, a lattice in $V$ is a free $\mathbb{k}[\epsilon]$-submodule of $V$, of rank $D$. Let $\mathscr{F}^{n}$ denote the set of $n$-step periodic lattices in $V$, that is, $\mathscr{F}^{n}$ consists of sequences of lattices $\boldsymbol{L}=\left(L_{i}\right)_{i \in \mathbb{Z}}$ where $L_{i-1} \subset L_{i}$, and $L_{i-n}=\epsilon L_{i}$ for all $i \in \mathbb{Z}$. We will also write $\mathscr{F}_{D}^{n}$ if we wish to emphasise the rank of $V$. Throughout this paper, if $X$ is a finite set, we will write $|X|$ for the cardinality of $X$.

The group $G=\operatorname{Aut}(V)$ of automorphisms of $V$ acts on $\mathscr{F}^{n}$ in the natural way. We shall be interested in functions supported on $\mathscr{F}^{n}$ and its square which are invariant with respect to the action of $G$ (where $G$ acts diagonally on $\mathscr{F}^{n} \times \mathscr{F}^{n}$ ). Thus we first describe the orbits of $G$ on these spaces. Let $\mathfrak{S}_{D, n}$ be the finite set of all $\boldsymbol{a}=\left(a_{i}\right)_{i \in \mathbb{Z}}$ such that

- $a_{i} \in \mathbb{N}$

- $a_{i}=a_{i+n}$ for all $i \in \mathbb{Z}$;

- $a_{i}+a_{i+1}+\cdots+a_{i+n-1}=D$ for all $i \in \mathbb{Z}$.

For $\boldsymbol{L} \in \mathscr{F}^{n}$, let $|\boldsymbol{L}| \in \mathfrak{S}_{D, n}$ be given by $|\boldsymbol{L}|_{i}=\operatorname{dim}\left(L_{i} / L_{i-1}\right)$. The $G$-orbits on $\mathscr{F}^{n}$ are indexed by this graded dimension: for $\boldsymbol{a} \in \mathfrak{S}_{D, n}$ set $\mathscr{F}_{\boldsymbol{a}}=\left\{\boldsymbol{L} \in \mathscr{F}^{n}:|\boldsymbol{L}|=\boldsymbol{a}\right\}$; then the $\mathscr{F}_{\boldsymbol{a}}$ are precisely the $G$-orbits on $\mathscr{F}^{n}$. The $G$-orbits on $\mathscr{F}^{n} \times \mathscr{F}^{n}$ are indexed, slightly more elaborately, by the set $\mathfrak{S}_{D, n, n}$ of matrices $A=\left(a_{i, j}\right)_{i, j \in \mathbb{Z}}$ such that

- $a_{i, j} \in \mathbb{N}$;

- $a_{i, j}=a_{i+n, j+n}$ for all $i, j \in \mathbb{Z}$;

- $a_{i, *}+a_{i+1, *}+\cdots+a_{i+n-1, *}=D$ for any $i \in \mathbb{Z}$;

- $a_{*, j}+a_{*, j+1}+\cdots+a_{*, j+n-1}=D$ for any $j \in \mathbb{Z}$. 
Here

$$
a_{i, *}=\sum_{j \in \mathbb{Z}} a_{i, j} \quad \text { and } \quad a_{*, j}=\sum_{i \in \mathbb{Z}} a_{i, j}
$$

For $A \in \mathfrak{S}_{D, n, n}$ set

$$
r(A)=\left(a_{i, *}\right)_{i \in \mathbb{Z}} \in \mathfrak{S}_{D, n} \quad \text { and } \quad c(A)=\left(a_{*, j}\right)_{j \in \mathbb{Z}} \in \mathfrak{S}_{D, n} .
$$

For $A \in \mathfrak{S}_{D, n, n}$ the corresponding $G$-orbit $\mathfrak{O}_{A}$ consists of pairs $\left(\boldsymbol{L}, \boldsymbol{L}^{\prime}\right)$ such that

$$
a_{i, j}=\operatorname{dim}\left(\frac{L_{i} \cap L_{j}^{\prime}}{\left(L_{i-1} \cap L_{j}^{\prime}\right)+\left(L_{i} \cap L_{j-1}^{\prime}\right)}\right),
$$

thus $\boldsymbol{L} \in \mathscr{F}_{r(A)}$ and $\boldsymbol{L}^{\prime} \in \mathscr{F}_{c(A)}$.

Let $\mathfrak{A}_{D ; q}$ be the space of integer-valued $G$-invariant functions on $\mathscr{F}^{n} \times \mathscr{F}^{n}$ supported on a finite number of orbits. If $e_{A}$ denotes the characteristic function of an orbit $\mathfrak{O}_{A}$, the set $\left\{e_{A}: A \in \mathfrak{S}_{D, n, n}\right\}$ is a basis of $\mathfrak{A}_{D ; q}$. The space $\mathfrak{A}_{D ; q}$ has a natural convolution product which gives it the structure of an associative algebra. With respect to the basis of characteristic functions the structure constants are given as follows. For $A, B, C \in \mathfrak{S}_{D, n, n}$, let $\eta_{A, B ; q}^{C}$ be the coefficient of $e_{C}$ in the product $e_{A} e_{B}$. Then $\eta_{A, B ; q}^{C}$ is zero unless $c(A)=r(B), r(A)=r(C)$ and $c(B)=c(C)$. Now suppose these conditions are satisfied and fix $\left(\boldsymbol{L}, \boldsymbol{L}^{\prime \prime}\right) \in \mathrm{O}_{C}$. Then $\eta_{A, B ; q}^{C}$ is the number of points in the set

$$
\left\{\boldsymbol{L}^{\prime} \in \mathscr{F}_{C(A))}:\left(\boldsymbol{L}, \boldsymbol{L}^{\prime}\right) \in \mathscr{O}_{A},\left(\boldsymbol{L}^{\prime}, \boldsymbol{L}^{\prime \prime}\right) \in \mathscr{O}_{B}\right\} .
$$

Clearly this is independent of the choice of $\left(\boldsymbol{L}, \boldsymbol{L}^{\prime \prime}\right)$, and moreover it can be shown that these structure constants are polynomial in $q$, allowing us to construct an algebra $\mathfrak{A}_{D, \mathbb{Z}[t]}$ over $\mathbb{Z}[t]$, which is a free $\mathbb{Z}[t]$-module on a basis $\left\{e_{A}: A \in \mathfrak{S}_{D, n, n}\right\}$ such that $\mathfrak{A}_{D, \mathbb{Z}[t] \mid t=q}=\mathfrak{A}_{D, q}$. In fact we will use the algebra $\mathfrak{A}_{D, \mathscr{A}}$ which is obtained from $\mathfrak{A}_{D, \mathbb{Z}[t]}$ by extending scalars to $\mathscr{A}=\mathbb{Z}\left[v, v^{-1}\right]$ where $v^{2}=t$ and the $\mathbb{Q}(v)$ algebra $\mathfrak{A}_{D}$ obtained by extending scalars to $\mathbb{Q}(v)$ (we will, by deliberate misuse, treat $v$ as both an indeterminate and a square root of $q$, depending on the context). The algebra $\mathfrak{A}_{D}$ is sometimes known as the affine $q$-Schur algebra. In what follows it will be more convenient to use a rescaled version of the basis $\left\{e_{A}\right\}_{A \in \mathfrak{S}_{D, n, n}}$ of $\mathfrak{A}_{D, \mathscr{A}}$, with elements $[A]=v^{-d_{A}} e_{A}$, where

$$
d_{A}=\sum_{\substack{i \geq k \\ j<l \\ 1 \leq i \leq n}} a_{i j} a_{k l}
$$

Note that if we define $\Psi([A])=\left[A^{t}\right]$, where $\left(A^{t}\right)_{i j}=a_{j i}$, then it is straightforward to check that $\Psi$ is an algebra antiautomorphism (see [Lusztig 1999, Lemma 1.11] for details), which we will sometimes call the transpose antiautomorphism. 
Next we introduce quantum groups. In order to do this we recall the notion of a root datum from [Lusztig 1993].

Definition 2.1. A Cartan datum is a pair $(I, \cdot)$ consisting of a finite set $I$ and a $\mathbb{Z}$-valued symmetric bilinear pairing on the free abelian group $\mathbb{Z}[I]$, such that

- $i \cdot i \in\{2,4,6, \ldots\}$ and

- $2 \frac{i \cdot j}{i \cdot i} \in\{0,-1,-2, \ldots\}$, for $i \neq j$.

A root datum of type $(I, \cdot)$ is a pair $Y, X$ of finitely generated free abelian groups and a perfect pairing $\langle\cdot, \cdot\rangle: Y \times X \rightarrow \mathbb{Z}$, together with embeddings $I \subset X(i \mapsto i)$ and $I \subset Y\left(i \mapsto i^{\prime}\right)$ such that $\left\langle i^{\prime}, j\right\rangle=2(i \cdot j) /(i \cdot i)$.

Given a root datum, we may define an associated quantum group $\mathbf{U}$. Since it is the only case we need, we will assume that our datum is symmetric and simply laced so that $i \cdot i=2$ for each $i \in I$, and $i \cdot j \in\{0,-1\}$ if $i \neq j$. In this case, $\mathbf{U}$ is generated as an algebra over $\mathbb{Q}(v)$ by symbols $E_{i}, F_{i}, K_{\mu}, i \in I, \mu \in Y$, subject to the following relations.

(1) $K_{0}=1, K_{\mu_{1}} K_{\mu_{2}}=K_{\mu_{1}+\mu_{2}}$ for $\mu_{1}, \mu_{2} \in Y$.

(2) $K_{\mu} E_{i} K_{\mu}^{-1}=v^{\left\langle\mu, i^{\prime}\right\rangle} E_{i}, \quad K_{\mu} F_{i} K_{\mu}^{-1}=v^{-\left\langle\mu, i^{\prime}\right\rangle} F_{i}$ for all $i \in I, \mu \in Y$.

(3) $E_{i} F_{j}-F_{j} E_{i}=\delta_{i, j}\left(K_{i}-K_{i}^{-1}\right) /\left(v-v^{-1}\right)$.

(4) $E_{i} E_{j}=E_{j} E_{i}, \quad F_{i} F_{j}=F_{j} F_{i}$, for $i, j \in I$ with $i \cdot j=0$.

(5) $E_{i}^{2} E_{j}+\left(v+v^{-1}\right) E_{i} E_{j} E_{i}+E_{j} E_{i}^{2}=0$ for $i, j \in I$ with $i \cdot j=-1$.

(6) $F_{i}^{2} F_{j}+\left(v+v^{-1}\right) F_{i} F_{j} F_{i}+F_{j} F_{i}^{2}=0$ for $i, j \in I$ with $i \cdot j=-1$.

Thus $\mathbf{U}$ is naturally $X$-graded, $\mathbf{U}=\bigoplus_{\nu \in X} \mathbf{U}_{v}$. The subalgebras $\mathbf{U}^{+}$and $\mathbf{U}^{-}$generated by the $E_{i}$ s and $F_{i}$ s respectively are isomorphic to each other, and indeed are isomorphic to the $\mathbb{Q}(v)$-algebra $\mathbf{f}$ generated by symbols $\left\{\theta_{i}: i \in I\right\}$ subject only to the relation

$$
\begin{aligned}
\theta_{i} \theta_{j}-\theta_{j} \theta_{i} & =0 \text { for } i, j \in I \text { with } i \cdot j=0, \\
\theta_{i}^{2} \theta_{j}+\left(v+v^{-1}\right) \theta_{i} \theta_{j} \theta_{i}+\theta_{j} \theta_{i}^{2} & =0 \text { for } i, j \in I \text { with } i \cdot j=-1 .
\end{aligned}
$$

Note that the algebra $\mathbf{f}$ depends only on the Cartan datum.

We also need to consider the modified quantum group $\dot{\mathbf{U}}$. This is defined by

$$
\dot{\mathbf{U}}=\bigoplus_{\lambda \in X} \mathbf{U} 1_{\lambda}, \quad \mathbf{U} 1_{\lambda}=\mathbf{U} / \sum_{\mu \in Y} \mathbf{U}\left(K_{\mu}-v^{\langle\mu, \lambda\rangle}\right),
$$

where the multiplicative structure is given in the natural way by the formulae

$$
\begin{aligned}
1_{\lambda} x & =x 1_{\lambda-v} \quad \text { for } x \in \mathbf{U}_{\nu}, \\
1_{\lambda} 1_{\lambda^{\prime}} & =\delta_{\lambda, \lambda^{\prime}} 1_{\lambda} .
\end{aligned}
$$


Let $\dot{\mathbf{U}}_{\mathscr{A}}$ be the $\mathscr{A}$-subalgebra of $\dot{\mathbf{U}}$ generated by $\left\{E_{i}^{(n)} 1_{\lambda}, F_{i}^{(n)} 1_{\lambda}: n \in \mathbb{Z}_{\geq 0}, \lambda \in X\right\}$. It is known [Lusztig 1993] that $\dot{\mathbf{U}}_{\mathscr{A}}$ is an $\mathscr{A}$-form of $\dot{\mathbf{U}}$, and moreover it is a free A-module.

To describe the connection between our convolution algebra and quantum groups, we will need the following notation. For $\boldsymbol{a} \in \mathfrak{S}_{D, n}$ let $\boldsymbol{i}_{\boldsymbol{a}} \in \mathfrak{S}_{D, n, n}$ be the diagonal matrix with $\left(\boldsymbol{i}_{\boldsymbol{a}}\right)_{i, j}=\delta_{i, j} a_{i}$. Let $E^{i, j} \in \mathfrak{S}_{1, n, n}$ be the matrix with $\left(E^{i, j}\right)_{k, l}$ equal to 1 if $k=i+s n$ and $l=j+s n$ for some $s \in \mathbb{Z}$, and to 0 otherwise. Let $\mathfrak{S}^{n}$ be the set of all $\boldsymbol{b}=\left(b_{i}\right)_{i \in \mathbb{Z}}$ such that $b_{i}=b_{i+n}$ for all $i \in \mathbb{Z}$. Let $\mathfrak{S}^{n, n}$ denote the set of all matrices $A=\left(a_{i, j}\right), i, j \in \mathbb{Z}$, with entries in $\mathbb{Z}$ such that

- $a_{i, j} \geq 0$ for all $i \neq j$

- $a_{i, j}=a_{i+n, j+n}$ for all $i, j \in \mathbb{Z}$;

- for any $i \in \mathbb{Z}$ the set $\left\{j \in \mathbb{Z}: a_{i, j} \neq 0\right\}$ is finite;

- for any $j \in \mathbb{Z}$ the set $\left\{i \in \mathbb{Z}: a_{i, j} \neq 0\right\}$ is finite.

Thus we have $\mathfrak{S}_{D, n, n} \subset \mathfrak{S}^{n, n}$ for all $D$. For $i \in \mathbb{Z} / n \mathbb{Z}$, let $\boldsymbol{i} \in \mathfrak{S}^{n}$ be given by $\boldsymbol{i}_{k}=1$ if $k=i \bmod n, \boldsymbol{i}_{k}=-1$ if $k=i+1 \bmod n$, and $\boldsymbol{i}_{k}=0$ otherwise. We write $\boldsymbol{a} \cup_{i} \boldsymbol{a}^{\prime}$ if $\boldsymbol{a}=\boldsymbol{a}^{\prime}+\boldsymbol{i}$. For such $\boldsymbol{a}, \boldsymbol{a}^{\prime}$ set

$$
\begin{aligned}
& { }_{\boldsymbol{a}} \boldsymbol{e}_{\boldsymbol{a}^{\prime}}=\boldsymbol{i}_{\boldsymbol{a}}-E^{i, i}+E^{i, i+1} \in \mathfrak{S}^{n, n}, \\
& { }_{\boldsymbol{a}^{\prime}} \mathbf{f}_{\boldsymbol{a}}=\boldsymbol{i}_{\boldsymbol{a}^{\prime}}-E^{i+1, i+1}+E^{i+1, i} \in \mathfrak{S}^{n, n} .
\end{aligned}
$$

Note that if $\boldsymbol{a}, \boldsymbol{a}^{\prime} \in \mathfrak{S}_{D, n}$ then ${ }_{\boldsymbol{a}} \boldsymbol{e}_{\boldsymbol{a}^{\prime}}, \boldsymbol{a}^{\prime} \mathbf{f}_{\boldsymbol{a}} \in \mathfrak{S}_{D, n, n}$. For $i \in \mathbb{Z} / n \mathbb{Z}$ set

$$
E_{i}(D)=\sum\left[{ }_{a} e_{a^{\prime}}\right], \quad F_{i}(D)=\sum\left[{ }_{a^{\prime}} \mathbf{f}_{\mathbf{a}}\right],
$$

where the sum is taken over all $\boldsymbol{a}, \boldsymbol{a}^{\prime}$ in $\mathfrak{S}_{D, n}$ such that $\boldsymbol{a} \cup_{i} \boldsymbol{a}^{\prime}$. For $\boldsymbol{a} \in \mathfrak{S}^{n}$ set

$$
K_{\boldsymbol{a}}(D)=\sum_{\boldsymbol{b} \in \mathfrak{S}_{D, n}} v^{\boldsymbol{a} \cdot \boldsymbol{b}}\left[\boldsymbol{i}_{\boldsymbol{b}}\right]
$$

where $\boldsymbol{a} \cdot \boldsymbol{b}=\sum_{i=1}^{n} a_{i} b_{i} \in \mathbb{Z}$ for any $\boldsymbol{a}, \boldsymbol{b} \in \mathfrak{S}^{n}$. If we let $X=Y=\mathfrak{S}^{n}$, and $I=\mathbb{Z} / n \mathbb{Z}$, with the embedding of $I \subset X=Y$ given by $i \mapsto i$ and pairing as given above, we obtain a symmetric simply laced root datum. We call the quantum group associated to it $\mathbf{U}\left(\widehat{\mathfrak{g l}}_{n}\right)$ (in fact this is the degenerate, or level zero, form of the affine quantum group associated to $\widehat{\mathfrak{g l}}_{n}$ ). It can be shown [Lusztig 1999] that the elements $E_{i}(D), F_{i}(D), K_{\boldsymbol{a}}(D)$ generate a subalgebra $\mathbf{U}_{D}$ which is a quotient of the quantum group $\mathbf{U}\left(\widehat{\mathfrak{g l}}_{n}\right)$, via the map that the notation suggests. In particular this gives the algebra $\mathfrak{A}_{D}$ the structure of a $\mathbf{U}\left(\widehat{\mathfrak{g l}}_{n}\right)$-module. Since $E_{i}(D), F_{i}(D), K_{\boldsymbol{a}}(D)$ all lie in $\mathfrak{A}_{D, \mathscr{A}}$ we similarly have an $\mathscr{A}$-subalgebra $\mathbf{U}_{D, \mathscr{A}}$. Note that the idea that one may extend the construction of [Beilinson et al. 1990] to the affine context is mentioned already in [Lusztig 1993, Notes on Part IV] and [Ginzburg and Vasserot 1993], but 
note that the surjectivity claimed in Theorem 9.2 of [Ginzburg and Vasserot 1993] is false, as shown in [Lusztig 1999].

Similarly, letting $\boldsymbol{b}_{0}=(\ldots, 1,1, \ldots) \in \mathfrak{S}^{n}$, we have a root datum given by $X^{\prime}=\mathfrak{S}^{n} / \mathbb{Z} \boldsymbol{b}_{0}, Y^{\prime}=\left\{\boldsymbol{a} \in \mathfrak{S}^{n}: \boldsymbol{a} \cdot \boldsymbol{b}_{0}=0\right\}$ with embeddings $I \subset X^{\prime}, Y^{\prime}$ induced by the above embeddings into $X$ and $Y$. This new root datum is associated to (again the degenerate form of) the quantum group $\mathbf{U}\left(\widehat{\mathfrak{s l}}_{n}\right)$. We have an algebra map $\phi_{D}: \dot{\mathbf{U}}\left(\widehat{\mathfrak{s l}}_{n}\right) \rightarrow \mathfrak{A}_{D}$ given by $E_{i}^{(n)} 1_{\lambda} \mapsto E_{i}(D)^{(n)}\left[\boldsymbol{i}_{\boldsymbol{a}}\right]$ where $\boldsymbol{a} \in \mathfrak{S}_{D, n}$ satisfies $\boldsymbol{a} \equiv \lambda \bmod \mathbb{Z} \boldsymbol{b}_{0}$ if such an $\boldsymbol{a}$ exists, and $E_{i}^{(n)} 1_{\lambda} \mapsto 0$ otherwise, and similarly for the $F_{i}^{(n)} 1_{\lambda}$. Clearly $\phi_{D}$ restricts to a map between the integral forms $\dot{\mathbf{U}}\left(\widehat{\mathfrak{s l}}_{n}\right)_{\mathscr{A}}$ and $\mathfrak{A}_{D, \mathscr{A}}$. It can be readily checked, using a Vandermonde determinant argument, that the image of $\phi_{D}$ is exactly $\mathbf{U}_{D}$ (see [Lusztig 1999, Lemma 2.8]).

\section{Inner product on $\mathbf{U}_{D}$}

Definition 3.1. We define a bilinear form

$$
(\cdot, \cdot)_{D}: \mathfrak{A}_{D ; q} \times \mathfrak{A}_{D ; q} \rightarrow \overline{\mathbb{Q}}_{l}
$$

by

$$
(f, \tilde{f})_{D}=\sum_{\boldsymbol{L}, \boldsymbol{L}^{\prime}} v^{\sum|\boldsymbol{L}|_{i}^{2}-\sum\left|\boldsymbol{L}^{\prime}\right|_{i}^{2}} f\left(\boldsymbol{L}, \boldsymbol{L}^{\prime}\right) \tilde{f}\left(\boldsymbol{L}, \boldsymbol{L}^{\prime}\right)
$$

for $f$ and $\tilde{f}$ in $\mathfrak{A}_{D, q}$, where $\boldsymbol{L}$ runs over $\mathscr{F}^{n}$ and $\boldsymbol{L}^{\prime}$ runs over a set of representatives for the $G$-orbits on $\mathscr{F}^{n}$.

Let $\mathrm{O}_{A}$ be a G-orbit on $\mathscr{F}^{n} \times \mathscr{F}^{n}$, and let

$$
X_{A}^{\boldsymbol{L}}=\left\{\boldsymbol{L}^{\prime} \in \mathscr{F}^{n}:\left(\boldsymbol{L}, \boldsymbol{L}^{\prime}\right) \in \mathcal{O}_{A}\right\}
$$

It is easy to check that

$$
2 d_{A}-2 d_{A^{t}}=\sum_{i=1}^{n} a_{i, *}^{2}-\sum_{j=1}^{n} a_{*, j}^{2} .
$$

Thus if $A, A^{\prime}$ are in $\mathfrak{S}_{D, n, n}$ we find that

$$
\left(e_{A}, e_{A^{\prime}}\right)_{D}=\delta_{A, A^{\prime}} q^{d_{A}-d_{A^{t}}}\left|X_{A^{t}}^{L^{\prime}}\right|,
$$

where $\boldsymbol{L}^{\prime}$ is any lattice in $\mathscr{F}_{C(A)}$. Thus the basis $\left\{e_{A}: A \in \mathfrak{S}_{D, n, n}\right\}$ is orthogonal for our inner product, and hence $(\cdot, \cdot)$ is nondegenerate. If $\left\{\eta_{A, B ; q}^{C}\right\}$ are the structure constants of $\mathfrak{A}_{D ; q}$ with respect to the basis $\left\{[A]: A \in \mathfrak{S}_{D, n, n}\right\}$, then we have

$$
\left([A],\left[A^{\prime}\right]\right)_{D}=\delta_{A, A^{\prime}} v^{-d_{A^{t}}} \eta_{A^{t}, A ; q}^{i_{c(A)}}
$$


We therefore obtain an inner product on $\mathfrak{A}_{D, \mathscr{A}}$ taking values in $\mathscr{A}$ by defining

$$
\left([A],\left[A^{\prime}\right]\right)_{D}=\delta_{A, A^{\prime}} v^{-d_{A^{t}}} \eta_{A^{t}, A}^{i_{c(A)}} \in \mathbb{Z}\left[v, v^{-1}\right] .
$$

By extending scalars, we obtain a $\mathbb{Q}(v)$-valued symmetric bilinear form on $\mathfrak{A}_{D}$. We now give some basic properties of this inner product:

Proposition 3.2. Let $A \in \mathfrak{S}_{D, n}$, and let $f, \tilde{f} \in \mathfrak{A}_{D}$. Then

$$
([A] f, \tilde{f})_{D}=v^{d_{A}-d_{A^{t}}}\left(f,\left[A^{t}\right] \tilde{f}\right) .
$$

Proof. Clearly it suffices to establish this equation in the algebra $\mathfrak{A}_{D ; q}$. Since the characteristic functions of G-orbits form a basis of $\mathfrak{A}_{D ; q}$, we may assume that $f=e_{B}$ and $\tilde{f}=e_{C}$, moreover we may assume that

$$
r(A)=r(C), \quad c(A)=r(B), \quad c(B)=c(C) .
$$

as both sides are zero otherwise. It follows immediately that

$$
[A] \cdot e_{B}=v^{-d_{A}} e_{A} \cdot e_{B}, \quad v^{d_{A}-d_{A^{t}}}\left[A^{t}\right] \cdot e_{C}=v^{d_{A}-2 d_{A^{t}}} e_{A^{t}} \cdot e_{C} .
$$

Hence, if $\left(\tilde{\boldsymbol{L}}, \boldsymbol{L}^{\prime}\right) \in \mathbb{O}_{C}$ is fixed,

$$
\begin{aligned}
\left([A] \cdot e_{B}, e_{C}\right)_{D} & =q^{d_{C}-d_{C^{t}}}\left|X_{C^{t}}^{\boldsymbol{L}^{\prime}}\right| \cdot v^{-d_{A}}\left|\left\{\boldsymbol{L}^{\prime \prime}:\left(\tilde{\boldsymbol{L}}, \boldsymbol{L}^{\prime \prime}\right) \in \mathcal{O}_{A},\left(\boldsymbol{L}^{\prime \prime}, \boldsymbol{L}^{\prime}\right) \in \mathcal{O}_{B}\right\}\right| \\
& =v^{\alpha}\left|\left\{\boldsymbol{L}, \boldsymbol{L}^{\prime \prime}:\left(\boldsymbol{L}, \boldsymbol{L}^{\prime \prime}\right) \in \mathbb{O}_{A},\left(\boldsymbol{L}^{\prime \prime}, \boldsymbol{L}^{\prime}\right) \in \mathbb{O}_{B},\left(\boldsymbol{L}, \boldsymbol{L}^{\prime}\right) \in \mathfrak{O}_{C}\right\}\right|,
\end{aligned}
$$

where $\alpha=2 d_{C}-2 d_{C^{t}}-d_{A}$. Similarly, if $\left(\tilde{\boldsymbol{L}}^{\prime \prime}, \boldsymbol{L}^{\prime}\right) \in \mathcal{O}_{B}$ is fixed,

$$
\begin{aligned}
& v^{d_{A}-d_{A^{t}}}\left(e_{B},\left[A^{t}\right] \cdot e_{C}\right)_{D} \\
& =q^{d_{B}-d_{B^{t}}}\left|X_{B^{t}}^{\boldsymbol{L}^{\prime}}\right| \cdot v^{d_{A}-2 d_{A^{t}}}\left|\left\{\boldsymbol{L}:\left(\tilde{\boldsymbol{L}}^{\prime \prime}, \boldsymbol{L}\right) \in \mathcal{O}_{A^{t}},\left(\boldsymbol{L}, \boldsymbol{L}^{\prime}\right) \in \mathcal{O}_{C}\right\}\right| \\
& =v^{\beta}\left|\left\{\boldsymbol{L}, \boldsymbol{L}^{\prime \prime}:\left(\boldsymbol{L}^{\prime \prime}, \boldsymbol{L}\right) \in \mathcal{O}_{A^{t}},\left(\boldsymbol{L}^{\prime \prime}, \boldsymbol{L}^{\prime}\right) \in \mathfrak{O}_{B},\left(\boldsymbol{L}, \boldsymbol{L}^{\prime}\right) \in \mathbb{O}_{C}\right\}\right| \text {, }
\end{aligned}
$$

where $\beta=2 d_{B}-2 d_{B^{t}}+d_{A}-2 d_{A^{t}}$. But now considering the diagrams

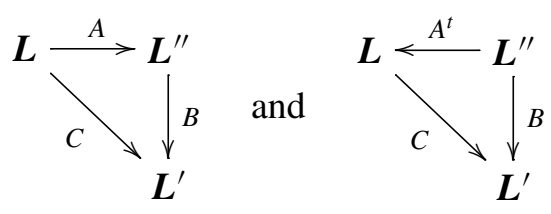

it is clear that the last line of (3-6) is the same as the last line of (3-7) if $\alpha=\beta$, that is, if

$$
2 d_{C}-2 d_{C^{t}}-d_{A}=2 d_{B}-2 d_{B^{t}}+d_{A}-2 d_{A^{t}}
$$

But this follows directly from (3-2) and (3-5).

We have the following easy consequence: 
Corollary 3.3. Let $i \in \mathbb{Z}$, and let $f, \tilde{f} \in \mathfrak{A}_{D}$ and $\boldsymbol{c} \in \mathfrak{S}^{n}$. Then

(1) $\left(E_{i}(f), \tilde{f}\right)_{D}=\left(f, v K_{i} F_{i}(\tilde{f})\right)_{D}$,

(2) $\left(F_{i}(f), \tilde{f}\right)_{D}=\left(f, v K_{-i} E_{i}(\tilde{f})\right)_{D}$,

(3) $\left(K_{\boldsymbol{c}}(f), \tilde{f}\right)_{D}=\left(f, K_{\boldsymbol{c}}(\tilde{f})\right)_{D}$.

Proof. We may assume that $f=e_{A}$ and $\tilde{f}=e_{B}$. The third equation can then be checked immediately from the formulas above. The second equation follows from the symmetry of the inner product and the other two, so it only remains to prove the first. We may assume that $r(A)=r(B)-\boldsymbol{i}$ and $c(A)=c(B)$, as both sides are zero otherwise. Set $\boldsymbol{a}=r(A), \boldsymbol{b}=r(B)$. Now from the definitions we have

$$
E_{i}\left(e_{A}\right)=\left[{ }_{b} \boldsymbol{e}_{\boldsymbol{a}}\right] \cdot e_{A}, \quad v K_{\boldsymbol{i}} F_{i}\left(e_{B}\right)=v^{1+\boldsymbol{i} \cdot \boldsymbol{a}}\left[{ }_{\boldsymbol{a}} \mathbf{f}_{\boldsymbol{b}}\right] \cdot e_{B} .
$$

Since ${ }_{b} \boldsymbol{e}_{\boldsymbol{a}}={ }_{\boldsymbol{a}} \mathbf{f}_{b}^{t}$ and $d_{b} \boldsymbol{e}_{a}-d_{a} \mathbf{f}_{b}=1+\boldsymbol{i} \cdot \boldsymbol{a}$, the result now follows immediately from the previous proposition.

Remark 3.4. There is a unique algebra antiautomorphism $\rho: \mathbf{U}\left(\widehat{\mathfrak{g l}}_{n}\right) \rightarrow \mathbf{U}\left(\widehat{\mathfrak{g l}}_{n}\right)$ such that

$$
\rho\left(E_{i}\right)=v K_{i} F_{i}, \quad \rho\left(F_{i}\right)=v K_{-i} E_{i} \quad \rho\left(K_{i}\right)=K_{i}
$$

With this we may state the result of the previous corollary in the form

$$
(u(f), \tilde{f})_{D}=(f, \rho(u) \tilde{f})_{D} \quad \text { for } u \in \mathbf{U}\left(\widehat{\mathfrak{g l}}_{n}\right) \text { and } f, \tilde{f} \in \mathfrak{A}_{D} .
$$

Note also that another natural choice ${ }^{1}$ of definition for an inner product on $\mathfrak{A}_{D, q}$ would be given by taking the sum in (3-1) over a set of representative of the $\operatorname{Aut}(V)$-orbits on $\mathscr{F}$ in the first factor, and all lattices in the second (the opposite of our choice). This inner product, which we denote by $(\cdot, \cdot)_{D}^{t}$ is obtained from the one we use via the transpose antiautomorphism $\Psi$, that is,

$$
(f, \tilde{f})_{D}^{t}=(\Psi(f), \Psi(\tilde{f}))_{D},
$$

and thus obeys "transposed" versions of the properties established in this section so that

$$
(f u, \tilde{f})_{D}^{t}=(f, \tilde{f} \bar{\rho}(u))_{D}^{t}, \quad u \in \mathbf{U}\left(\widehat{\mathfrak{g l}}_{n}\right), \quad f, \tilde{f} \in \mathfrak{A}_{D},
$$

where $\bar{\rho}$ is given by $\bar{\rho}(x)=\overline{\rho(\bar{x})}$. The precise relation between $(\cdot, \cdot)_{D}$ and $(\cdot, \cdot)_{D}^{t}$ can be given as follows: if $f, \tilde{f} \in\left[\boldsymbol{i}_{\boldsymbol{a}}\right] \mathfrak{A}_{D}\left[\boldsymbol{i}_{\boldsymbol{b}}\right]$ then

$$
v^{\sum_{i=1}^{n} b_{i}^{2}}(\llbracket \boldsymbol{b} \rrbracket !)^{-1}(f, \tilde{f})_{D}=v^{\sum_{i=1}^{n} a_{i}^{2}}(\llbracket \boldsymbol{a} \rrbracket !)^{-1}(f, \tilde{f})_{D}^{t} .
$$

where we define $\llbracket a \rrbracket !=\prod_{i=1}^{n} \prod_{j=1}^{a_{i}}\left(1-v^{-2 j}\right)$. In the finite type case of [Beilinson et al. 1990] this follows easily from considering the orbits on the product of the

\footnotetext{
${ }^{1}$ It is clear that one needs to restrict one factor to run over representatives of the $G$-orbits to avoid infinity sums — in the finite type case considered in [Beilinson et al. 1990] this issue doesn't arise.
} 
space of $n$-step flags with itself, while in the affine case it requires some more care. Since we will not use this fact we do not include the details.

Lemma 3.5. (1) For $A \in \mathfrak{S}_{D, n, n}$, we have $([A],[A])_{D} \in 1+v^{-1} \mathbb{Z}\left[v^{-1}\right]$.

(2) For $A, A^{\prime} \in \mathfrak{S}_{D, n, n}$ and $A \neq A^{\prime}$, we have $\left([A],\left[A^{\prime}\right]\right)_{D}=0$.

Proof. The second part of the statement is obvious. For the first, note that by [Lusztig 1999, 4.3] the set $X_{A^{t}}^{L^{\prime}}$ is an irreducible variety of dimension $d_{A^{t}}$. Since

$$
\left([A],\left[A^{\prime}\right]\right)_{D}=\delta_{A, A^{\prime}} q^{-d_{A^{t}}\left|X_{A^{t}}^{L^{\prime}}\right|,}
$$

the Lang-Weil estimates [1954] then show that $([A],[A])_{D}$ lies in $1+v^{-1} \mathbb{Z}\left[v^{-1}\right]$, as required.

Remark 3.6. The results of this section are analogues of the results of [Lusztig 1999, §7]; however our inner product is not the same as that of [Lusztig 1999, 7.1], and this makes the proofs somewhat simpler. Lusztig's choice of inner product is compatible with the left and right $\mathbf{U}$-module structure, in the sense that Equations (3-9) and (3-10) both hold. Although this is not quite proved in [Lusztig 1999] it follows from our above discussion using (3-11). However, it does not produce the inner product on $\dot{\mathbf{U}}$ in the way we need.

\section{Inner product on $\dot{U}$}

In this section we will write $\dot{\mathbf{U}}$ to denote the modified quantum group $\dot{\mathbf{U}}\left(\widehat{\mathfrak{s l}}_{n}\right)$ associated to the root datum $\left(X^{\prime}, Y^{\prime}\right)$ defined in Section 2. We wish to obtain an inner product on $\dot{\mathbf{U}}$ using those on the family of algebras $\left\{\mathbf{U}_{D}\right\}_{D \in \mathbb{N}}$.

We begin with some technical lemmas. Given $A \in \mathfrak{S}^{n, n}$ let $a_{i, \geq s}=\sum_{j \geq s} a_{i, j}$, and $a_{i,>s}, a_{i, \leq s}$, etc. similarly.

Lemma 4.1. (a) Let $A \in \mathfrak{S}_{D, n, n}$ and $\boldsymbol{a}^{\prime}=r(A)$. If there is an $\boldsymbol{a} \in \mathfrak{S}_{D, n}$ such that $\boldsymbol{a} \cup_{i} \boldsymbol{a}^{\prime}$ (i.e., if $a_{i+1}^{\prime}>0$ ), then

$$
\left[{ }_{\boldsymbol{a}} \boldsymbol{e}_{\boldsymbol{a}^{\prime}}\right][A]=\sum_{\substack{s \in \mathbb{Z} \\ a_{i+1, s} \geq 1}} v^{a_{i, \geq s}-a_{i+1,>s}}\left(\frac{1-v^{-2\left(a_{i, s}+1\right)}}{1-v^{-2}}\right)\left[A+E^{i, s}-E^{i+1, s}\right],
$$

where $A=\left(a_{i, j}\right)$.

(b) Let $A^{\prime} \in \mathfrak{S}_{D, n, n}$ and $\boldsymbol{a}=r\left(A^{\prime}\right)$. If there is an $\boldsymbol{a}^{\prime} \in \mathfrak{S}_{D, n}$ such that $\boldsymbol{a} \cup_{i} \boldsymbol{a}^{\prime}$ (i.e., if $\left.a_{i}>0\right)$, then

$$
\left[\boldsymbol{a}^{\prime} \mathbf{f}_{\boldsymbol{a}}\right]\left[A^{\prime}\right]=\sum_{\substack{s \in \mathbb{Z} \\ a_{i, s}^{\prime} \geq 1}} v^{a_{i+1, \leq s}^{\prime}-a_{i,<s}^{\prime}}\left(\frac{1-v^{-2\left(a_{i+1, s}^{\prime}+1\right)}}{1-v^{-2}}\right)\left[A^{\prime}-E^{i, s}+E^{i+1, s}\right],
$$

where $A^{\prime}=\left(a_{i, j}^{\prime}\right)$. 
Proof. This follows by rescaling the statement of [Lusztig 1999, Proposition 3.5].

Let $\mathscr{R}$ be the subring of $\mathbb{Q}(v)[u]$ generated by $\left\{v^{j}: j \in \mathbb{Z}\right\}$ and by the elements

$$
\prod_{i=1}^{t} \frac{v^{-2(a-i)} u^{2}-1}{v^{-2 i}-1} \text { for } a \in \mathbb{Z}, t \geq 1 .
$$

For $A \in \mathfrak{S}^{n, n}$ let ${ }_{p} A$ be the matrix with $\left(_{p} A\right)_{i, j}=a_{i, j}+p \delta_{i, j}$. We have the following partial analogue of [Beilinson et al. 1990, 4.2].

Lemma 4.2. Let $A_{1}, A_{2}, \ldots, A_{k}$ be matrices of the form $\boldsymbol{a}_{\boldsymbol{a}} \boldsymbol{a}^{\prime}$ or $\boldsymbol{f}_{\boldsymbol{a}} \mathbf{f}_{\boldsymbol{a}^{\prime}}$, for $\boldsymbol{a}, \boldsymbol{a}^{\prime} \in \mathfrak{S}^{n}$, and $A$ any element of $\mathfrak{S}^{n, n}$. Then there exist matrices $Z_{1}, Z_{2}, \ldots, Z_{m} \in \mathfrak{S}^{n, n}$, and $G_{1}, G_{2}, \ldots, G_{m} \in \mathscr{R}$ and an integer $p_{0} \in \mathbb{Z}$ such that

$$
\left[{ }_{p} A_{1}\right]\left[{ }_{p} A_{2}\right] \ldots\left[{ }_{p} A_{k}\right]\left[{ }_{p} A\right]=\sum_{i=1}^{m} G_{i}\left(v, v^{-p}\right)\left[{ }_{p} Z_{i}\right],
$$

for all $p \geq p_{0}$.

Proof. This follows using the same argument as in the proof of Proposition 4.2 in [Beilinson et al. 1990] (where a similar but stronger result is proved for the finite-type case). One uses induction on $k$. When $k=1$ the result follows from the previous lemma, once we note that both $a_{i, \geq s}-a_{i+1,>s}$ and $a_{i+1, \leq s}-a_{i,<s}$ are unchanged when $A$ is replaced with $A+p I$.

Recall from Section 2 that there is a surjective homomorphism $\phi_{D}: \dot{\mathbf{U}} \rightarrow \mathbf{U}_{D}$ which, for $\lambda \in X$, sends $E_{i} 1_{\lambda} \mapsto E_{i}(D)\left[\boldsymbol{i}_{\boldsymbol{a}}\right]$ and $F_{i} 1_{\lambda} \mapsto F_{i}(D)\left[\boldsymbol{i}_{\boldsymbol{a}}\right]$ if there is an $\boldsymbol{a}$ in $\mathfrak{S}_{D, n}$ such that $\boldsymbol{a}=\lambda \bmod \mathbb{Z} \boldsymbol{b}_{0}$, otherwise both $E_{i} 1_{\lambda}, F_{i} 1_{\lambda}$ are sent to zero. Let $\mathbf{f}$ be the algebra defined in Section 2. Pick a monomial basis of $\mathbf{f}$, $\left\{\zeta_{i}: i \in J\right\}$ say. The triangular decomposition for $\dot{\mathbf{U}}$ [Lusztig 1992, 23.2.1] shows that $\mathfrak{B}=\left\{\zeta_{i}^{+} \zeta_{j}^{-} 1_{\lambda}: i, j \in J, \lambda \in X\right\}$ is a basis of $\dot{\mathbf{U}}$, where $+: \mathbf{f} \rightarrow \mathbf{U}^{+}$, and - : $\mathbf{f} \rightarrow \mathbf{U}^{-}$are the standard isomorphisms defined by $\theta_{i} \mapsto E_{i}$ and $\theta_{i} \mapsto F_{i}$ respectively. Define a bilinear pairing $\langle\cdot, \cdot\rangle_{D}$ on $\dot{\mathbf{U}}$ via $\phi_{D}$ as follows:

$$
\langle x, y\rangle_{D}=\left(\phi_{D}(x), \phi_{D}(y)\right)_{D} .
$$

Proposition 4.3. Let $k \in\{0,1, \ldots, n-1\}$, then if $x, y \in \dot{\mathbf{U}}$

$$
\langle x, y\rangle_{k+p n}
$$

converges in $\mathbb{Q}\left(\left(v^{-1}\right)\right)$, as $p \rightarrow \infty$, to an element of $\mathbb{Q}(v)$.

Proof. We may assume that $x, y$ are elements of $\mathfrak{B}$. Then we need to show that

$$
\left\langle\zeta_{i_{1}}^{+} \zeta_{j_{1}}^{-} 1_{\lambda}, \zeta_{i_{2}}^{+} \zeta_{j_{2}}^{-} 1_{\mu}\right\rangle_{k+p n}, \quad \text { for } i_{1}, i_{2}, j_{1}, j_{2} \in J ; \lambda, \mu \in X,
$$


converges as $p \rightarrow \infty$. Let $\iota: \mathbf{f} \rightarrow \mathbf{f}$ be the $\mathbb{Q}(v)$-algebra antiautomorphism fixing the generators $\theta_{i}, 1 \leq i \leq n$. Using Corollary 3.3, it is easy to see that this inner product differs from

$$
\left\langle 1_{\lambda}, \iota\left(\zeta_{j_{1}}\right)^{+} \iota\left(\zeta_{i_{1}}\right)^{-} \zeta_{i_{2}}^{+} \zeta_{j_{2}}^{-} 1_{\mu}\right\rangle_{k+p n}
$$

by a power of $v$ which is independent of $p$. But then the definition of the inner product and Lemma 4.2 show that (4-4) may be written as $G\left(v, v^{-p}\right)$ for some $G \in \mathscr{R}$. The result then follows immediately from the definition of $\mathscr{R}$.

Remark 4.4. The proof of the last proposition actually allows us to conclude that

$$
\left(\phi_{D}\left(\zeta_{i}^{+} \zeta_{j}^{-} 1_{\lambda}\right),[p A]\right)_{k+p n}
$$

converges to an element of $\mathbb{Q}(v)$, as $p \rightarrow \infty$, for any $A \in \mathfrak{S}^{n, n}$. We will need this in the next section.

Definition 4.5. We define

$$
\langle\cdot, \cdot\rangle: \dot{\mathbf{U}} \times \dot{\mathbf{U}} \rightarrow \mathbb{Q}(v),
$$

a symmetric bilinear form on $\dot{\mathbf{U}}$ given by

$$
\langle x, y\rangle=\sum_{k=0}^{n-1} \lim _{p \rightarrow \infty}\langle x, y\rangle_{k+p n} .
$$

Remark 4.6. Although the inner products $(\cdot, \cdot)_{D}$ satisfy only (3-9) and not (3-10), the formula (3-11) which relates $(\cdot, \cdot)_{D}$ and $(\cdot, \cdot)_{D}^{t}$ can be used to show that our limiting inner product $\langle\cdot, \cdot\rangle$ satisfies the analogue of both equations, as indeed Lusztig shows for his inner product on $\dot{\mathbf{U}}$ in [Lusztig 1993, Proposition 26.1.3].

\section{Comparison of inner products}

Lusztig has shown that the algebra $\dot{\mathbf{U}}$ has a natural inner product which characterised by the following result. (Again, in this section $\dot{\mathbf{U}}$ denotes the modified quantum group attached to the root datum $\left(X^{\prime}, Y^{\prime}\right)$.)

Theorem 5.1 (Lusztig). There exists a unique $\mathbb{Q}(v)$ bilinear pairing

$$
(\cdot, \cdot): \dot{\mathbf{U}} \times \dot{\mathbf{U}} \rightarrow \mathbb{Q}(v)
$$

such that

(1) $\left(1_{\lambda_{1}} x 1_{\lambda_{2}}, 1_{\mu_{1}} y 1_{\mu_{2}}\right)=0$ for all $x, y \in \dot{\mathbf{U}}$ unless $\lambda_{1}=\mu_{1}, \lambda_{2}=\mu_{2}$;

(2) $(u x, y)=(x, \rho(u) y)$ for all $x, y \in \dot{\mathbf{U}}$ and $u \in \mathbf{U}$;

(3) $\left(x^{-} 1_{\lambda}, y^{-} 1_{\lambda}\right)=(x, y)$ for all $x, y \in \mathbf{f}$ and $\lambda \in X$. 
Here $(x, y)$ is the standard inner product on $\mathbf{f}$ (see [Lusztig 1993, 1.2.5]). The resulting inner product is automatically symmetric.

Proof. See [Lusztig 1993, 26.1.2].

Theorem 5.2. The inner products $\langle\cdot, \cdot\rangle$ of Section 4 and $(\cdot, \cdot)$ of Theorem 5.1 coincide.

The remainder of this section is devoted to a proof of this theorem. Property (1) in Theorem 5.1 clearly holds for $\langle\cdot, \cdot\rangle$, as the representatives for elements of $X$ in $\mathfrak{S}_{D, n}$ are distinct when they exist. Property (2) follows from Corollary 3.3; thus it only remains to verify (3).

Fix $\lambda \in X$. The algebra $\mathbf{f}$ is naturally graded: $\mathbf{f}=\bigoplus_{v \in \mathbb{N} I} \mathbf{f}_{v}$. For $v \in \mathbb{Z}[I]$ with $v=\sum_{i \in I} v_{i} i$, let $\operatorname{tr}(v)=\sum_{i \in I} v_{i}$. If $z$ is homogeneous we set $|z|=v$, where $z \in \mathbf{f}_{v}$. Thus for the third property we may assume that $x, y \in \mathbf{f}$ are homogeneous, i.e., $x, y \in \mathbf{f}_{v}$ for some $v$, and proceed by induction on $N=\operatorname{tr}(v)$. If $N=0$ then we are reduced to the equation

$$
\left\langle 1_{\lambda}, 1_{\lambda}\right\rangle=1
$$

which holds trivially. Now suppose that $N>0$ and the result is known for $x, y \in \mathbf{f}_{v}$ when $\operatorname{tr}(v)<N$. If $x, y$ are in $\mathbf{f}_{v}$ and $\operatorname{tr}(v)=N$, then we may assume that they are monomials, and $y=\theta_{i} z$ for some $z \in \mathbf{f}_{v-i}$. Thus we have

$$
\begin{aligned}
\left\langle x^{-} 1_{\lambda}, y^{-} 1_{\lambda}\right\rangle & =\left\langle x^{-} 1_{\lambda}, F_{i} z^{-} 1_{\lambda}\right\rangle \\
& =\left\langle v K_{-i} E_{i} x^{-} 1_{\lambda}, z^{-} 1_{\lambda}\right\rangle,
\end{aligned}
$$

using property (2) of the inner product (which we have already seen holds for both $(\cdot, \cdot)$ and $\langle\cdot, \cdot\rangle)$. Now using standard commutation formulas (see [Lusztig 1993, 3.1.6]) this becomes

$$
\left\langle v K_{-i} x^{-} E_{i} 1_{\lambda}, z^{-} 1_{\lambda}\right\rangle+\frac{1}{1-v^{-2}}\left\langle\left({ }_{i} r(x)^{-}-v K_{-i} r_{i}(x)^{-} K_{-i}\right) 1_{\lambda}, z^{-} 1_{\lambda}\right\rangle
$$

where ${ }_{i} r$ and $r_{i}$ are the twisted derivations defined in [Lusztig 1993, 1.2.13]. Tidying this up we get

$$
\frac{1}{1-v^{-2}}\left\langle{ }_{i} r(x)^{-} 1_{\lambda}, z^{-} 1_{\lambda}\right\rangle+\left\langle v^{i \cdot|x|-i \cdot \lambda-1}\left(x^{-} E_{i}-\frac{v^{-i \cdot \lambda}}{v-v^{-1}} r_{i}(x)^{-}\right) 1_{\lambda}, z^{-} 1_{\lambda}\right\rangle
$$

But ${ }_{i} r(x), z \in \mathbf{f}_{v-i}$, hence by induction we have $\left\langle{ }_{i} r(x) 1_{\lambda}, z 1_{\lambda}\right\rangle=\left({ }_{i} r(x)^{-}, z\right)$, and by standard properties of the inner product $(\cdot, \cdot)$ on $\mathbf{f}$ we know that

$$
\frac{1}{1-v^{-2}}\left({ }_{i} r(x), z\right)=\left(x, \theta_{i} z\right)
$$


thus we are done by induction if we can show that for any $x \in \mathbf{f}_{v}$ the element

$$
u(x)=\left(x^{-} E_{i}-\frac{v^{-i \cdot \lambda}}{v-v^{-1}} r_{i}(x)^{-}\right) 1_{\lambda}
$$

annihilates $\mathbf{U}^{-} 1_{\lambda}$. To see this we need some (rather technical) lemmas about multiplication in $\mathfrak{A}_{D}$.

Lemma 5.3. Let $A \in \mathfrak{S}^{n, n}$ be such that $a_{r, s}=0$ for $r<s$ unless $r=s-1$ and $r=i$ $\bmod n$, when $a_{r, r+1} \in\{0,1\}$; then the following hold for $p$ sufficiently large.

(1) For $j \neq i$ we have

$$
F_{j}\left[{ }_{p} A\right]=\sum_{k=1}^{m} g_{k}(v)\left[{ }_{p} Z_{k}\right]
$$

where $g_{k}(v) \in \mathscr{A}$ and $Z_{k} \in \mathfrak{S}^{n, n}(1 \leq k \leq m)$ and moreover $g_{k}(v)$ is independent both of $p$ and $\left\{a_{r, s}: r \leq s\right\}$, and we have $\left(Z_{k}\right)_{r, s}=a_{r, s}$ for $r<s$.

(2)

$$
\begin{aligned}
F_{i}\left[{ }_{p} A\right]=\sum_{k=1} & g_{k}(v)\left[{ }_{p} Z_{k}\right] \\
& +v^{1-i \cdot r(A)}\left(\frac{1-v^{-2\left(a_{i+1, i+1}+1+p\right)}}{1-v^{-2}}\right)\left[{ }_{p}\left(A+E^{i+1, i+1}-E^{i, i+1}\right)\right],
\end{aligned}
$$

where $g_{k}(v) \in \mathscr{A}$ is independent of $p$ and $\left\{a_{r, s}: r \leq s\right\}$, and we have $\left(Z_{k}\right)_{r, s}=a_{r, s}$ for $r<s$, and the final term occurs only if $a_{i, i+1}=1$.

Proof. By Lemma 4.1, for any $A \in \mathfrak{S}^{n, n}$ and $p$ large enough we have

$$
F_{j}\left[{ }_{p} A\right]=\sum_{k:\left({ }_{p} A\right)_{j, k} \geq 1} v^{a_{j+1, \leq k}-a_{j,<k}}\left(\frac{1-v^{-2\left(a_{j+1, k}+p \delta_{j+1, k}+1\right)}}{1-v^{-2}}\right)\left[{ }_{p} A+E^{j+1, k}-E^{j, k}\right] .
$$

We claim that in our case the coefficients are independent of $p$ and of $\left\{a_{r, s}: r \leq s\right\}$, unless $j \equiv i \bmod n$. Indeed then $\left({ }_{p} A\right)_{j, k} \geq 1$ implies that $j \geq k$, and hence the coefficient of $\left[{ }_{p} A+E^{j+1, k}-E^{j, k}\right]$ in the sum above is

$$
v^{a_{j+1, \leq k}-a_{j,<k}}\left(\frac{1-v^{-2\left(a_{j+1, k}+1\right)}}{1-v^{-2}}\right),
$$

which evidently involves only entries $a_{r, s}$ of $A$ with $r>s$, thus establishing the first part of the lemma.

For the second part, if $j \equiv i \bmod n$ then we get the same conclusion except when $k=j+1$, if $a_{j, j+1}=1$ in which case $a_{j+1, \leq j+1}=a_{j+1, *}$ and $a_{j,<j+1}=a_{j, *}-1$ by our assumptions, so that the term $a_{j+1, \leq k}-a_{j,<k}=1-\boldsymbol{i} \cdot r(A)$, and this yields the final term in second part, as required. 
Fix $\lambda \in X^{\prime}$. Then $\sum_{i=1}^{n} \lambda_{i}=k \bmod n$ for a well-defined $k \in\{0,1, \ldots n-1\}$. If $D=k+p n$, then there is a unique $\boldsymbol{a} \in X$ which is a representative of $\lambda \in X^{\prime}$ satisfying $\sum_{i=1}^{n} a_{i}=D$ (that is, $\boldsymbol{a} \in \mathfrak{S}_{D, n}$ ).

Lemma 5.4. Let $\sum_{j=1}^{n} \lambda_{j}=k \bmod n$, where $k \in\{0,1, \ldots, n-1\}$, and suppose that $D=k+p n$ for some $p$. Then if $x$ is a monomial in the generators $\left\{\theta_{i}: i \in I\right\}$ we have, for sufficiently large $p$,

$$
\begin{aligned}
\phi_{D}\left(x^{-} E_{i} 1_{\lambda}\right) & =\sum_{k=1}^{m_{1}} a_{k}(v)\left[{ }_{p} B_{k}\right]+\sum_{k=1}^{m_{2}}\left(b_{k}(v)+v^{-2 p} c_{k}(v)\right)\left[{ }_{p} H_{k}\right], \\
\phi_{D}\left(x^{-} 1_{\lambda}\right) & =\sum_{k=1}^{m_{1}} a_{k}(v)\left[{ }_{p} B_{k}+E^{i+1, i+1}-E^{i, i+1}\right]
\end{aligned}
$$

for some $B_{k}, H_{k} \in \mathfrak{S}^{n, n}$ independent of $p$, where $\left(B_{k}\right)_{r, s}=\left(H_{k}\right)_{r, s}=0$ for $r<s$ unless $r=i \bmod n$ when $\left(B_{k}\right)_{i, i+1}=1$, and the coefficients $a_{k}, b_{k}, c_{k}$ are independent of $p$, with $a_{k} \in \mathscr{A}$ and $b_{k}, c_{k} \in\left(v-v^{-1}\right)^{-1} \mathscr{A}$. Moreover, we have

$$
\frac{v^{-i \cdot \lambda}}{v-v^{-1}} \phi_{D}\left(r_{i}(x)^{-} 1_{\lambda}\right)=\sum_{k=1}^{m_{2}} b_{k}(v)\left[{ }_{p} H_{k}\right]
$$

Proof. We use induction on $N=\operatorname{tr}(|x|)$. If $N=0$ then the result is clear, since we have $\phi_{D}\left(E_{i} 1_{\lambda}\right)=\left[\boldsymbol{i}_{\boldsymbol{a}}+E^{i, i+1}-E^{i+1, i+1}\right]$ and $\phi_{E}\left(1_{\lambda}\right)=\left[\boldsymbol{i}_{\boldsymbol{a}}\right]$ (thus in this case we have $m_{1}=1$ and $m_{2}=0$ ). Now suppose the result is known for all $y$ with $\operatorname{tr}(|y|)<N$. We may write $x=\theta_{j} z$ where $\operatorname{tr}(|z|)=N-1$.

By induction, we have

$$
\begin{aligned}
\phi_{D}\left(x E_{i} 1_{\lambda}\right) & =F_{j} \phi_{D}\left(z E_{i} 1_{\lambda}\right) \\
& =F_{j}\left(\sum_{k=1}^{m_{1}^{\prime}} a_{k}^{\prime}(v)\left[{ }_{p} B_{k}^{\prime}\right]+\sum_{k=1}^{m_{2}^{\prime}}\left(b_{k}^{\prime}(v)+v^{-2 p} c_{k}^{\prime}(v)\right)\left[{ }_{p} H_{k}^{\prime}\right]\right),
\end{aligned}
$$

with $a_{k}^{\prime}, b_{k}^{\prime}, c_{k}^{\prime}, B_{k}^{\prime}, H_{k}^{\prime}$ as in the statement of the lemma (for $x=z$ ). Now applying Lemma 5.3 to each of these terms, we find that

$$
\begin{aligned}
& \phi_{D}\left(x E_{i} 1_{\lambda}\right)= \\
& \sum_{k=1}^{m_{1}} a_{k}(v)\left[{ }_{p} B_{k}\right]+\delta_{i, j} v^{1-i \cdot r\left(B_{k}^{\prime}\right)}\left(\frac{1-v^{-2\left(\left(B_{k}^{\prime}\right)_{i+1, i+1}+1+p\right)}}{1-v^{-2}}\right) a_{k}^{\prime}(v)\left[{ }_{p} B_{k}^{\prime}+E^{i+1, i+1}-E^{i, i+1}\right] \\
& +\sum_{k=1}^{m_{2}^{\prime \prime}}\left(b_{k}(v)+v^{-2 p} c_{k}\right)\left[{ }_{p} H_{k}\right], \quad \text { (5-3) }
\end{aligned}
$$

where $\left(B_{k}\right)_{r, s}=0$ if $r<s$ unless $r=i \bmod n$ and $s=r+1$, and similarly $\left(H_{k}\right)_{r, s}=0$ if $r<s$. Thus the first formula of the lemma is established by induction once we 
note that when $i \equiv j \bmod n$ we may write

$$
\begin{aligned}
v^{1-i \cdot r\left(B_{k}^{\prime}\right)}( & \left.\frac{1-v^{-2\left(\left(B_{k}^{\prime}\right)_{i+1, i+1}+1+p\right)}}{1-v^{-2}}\right) a_{k}^{\prime}(v)\left[{ }_{p} B_{k}^{\prime}+E^{i+1, i+1}-E^{i, i+1}\right] \\
& =\frac{v^{-i \cdot r\left(B_{k}^{\prime}\right)}}{v-v^{-1}}\left(1-v^{-2 p} v^{-2\left(\left(B_{k}^{\prime}\right)_{i+1, i+1}+1\right.}\right) a_{k}^{\prime}(v)\left[{ }_{p} B_{k}^{\prime}+E^{i+1, i+1}-E^{i, i+1}\right] \\
& =\left(b_{m_{2}^{\prime \prime}+k}+v^{-2 p} c_{m_{2}^{\prime \prime}+k}\right)\left[H_{m_{2}^{\prime \prime}+k}\right],
\end{aligned}
$$

where the last line defines $b_{m_{2}^{\prime \prime}+k}, c_{m_{2}^{\prime \prime}+k}$ and $H_{m_{2}^{\prime \prime}+k}$, and by induction $b_{m_{2}^{\prime \prime}+k}, c_{m_{2}^{\prime \prime}+k}$ lie in $\left(v-v^{-1}\right)^{-1} \mathscr{A}$, since $a_{k}^{\prime}(v) \in \mathscr{A}$. Setting $m_{2}=m_{2}^{\prime \prime}+\delta_{i, j} m_{1}$ the first formula is therefore established.

To show the second formula, we again use induction so that we have

$$
\phi_{D}\left(x^{-} 1_{\lambda}\right)=F_{j} \cdot \sum_{k=1}^{m_{1}^{\prime}} a_{k}^{\prime}(v)\left[{ }_{p} B_{k}^{\prime}+E^{i+1, i+1}-E^{i, i+1}\right]
$$

Now by Lemma 5.3 (in particular the independence of the coefficients from the values of $\left\{a_{r, s}: r \leq s\right\}$ in all but the final term of the second formula) this is equal to

$$
\sum_{k=1}^{m_{1}} a_{k}(v)\left[{ }_{p} B_{k}+E^{i+1, i+1}-E^{i, i+1}\right],
$$

as required. Finally, to see the "moreover" part of the lemma, note that by definition we have

$$
\begin{aligned}
\left(\frac{v^{-i \cdot \lambda}}{v-v^{-1}}\right) \phi_{D}\left(r_{i}(x)^{-} 1_{\lambda}\right) & =\left(\frac{v^{-i \cdot \lambda}}{v-v^{-1}}\right) \phi_{D}\left(r_{i}\left(\theta_{j} z\right)^{-} 1_{\lambda}\right) \\
& =\left(\frac{v^{-i \cdot \lambda}}{v-v^{-1}}\right)\left(\delta_{i, j} v^{i \cdot|z|} \phi_{D}\left(z^{-} 1_{\lambda}\right)+F_{j}\left(\phi_{D}\left(r_{i}(z)^{-} 1_{\lambda}\right)\right) .\right.
\end{aligned}
$$

Comparing this with (5-3), we note that $r\left(B_{k}^{\prime}\right)=\boldsymbol{a}+\boldsymbol{i}-|z|$, hence $1-\boldsymbol{i} \cdot r\left(B_{k}^{\prime}\right)=$ $i \cdot(|z|-\lambda)-1$, so that

$$
v^{1-i \cdot r\left(B_{k}^{\prime}\right)}\left(\frac{1-v^{-2\left(\left(B_{k}^{\prime}\right)_{i+1, i+1}+p+1\right)}}{1-v^{-2}}\right)=\left(\frac{v^{i \cdot(|z|-\lambda)}}{v-v^{-1}}\right)\left(1-v^{-2 p} v^{-2\left(\left(B_{k}^{\prime}\right)_{i+1, i+1}+1\right)}\right) .
$$

Hence the result follow once again by induction.

Having established these technical lemmas, it is now straightforward to complete the proof of Theorem 5.2.

Definition 5.5. Let

$$
\mathfrak{A}_{D}^{-}=\operatorname{span}\left\{[A]: a_{r, s}=0 \text { for all } r<s\right\}
$$


and let $\pi_{D}: \mathfrak{A}_{D} \rightarrow \mathfrak{A}_{D}^{-}$be the orthogonal projection; thus its kernel is spanned by the elements $[A]$ such that $a_{r, s}>0$ for some $r, s \in \mathbb{Z}$ with $r<s$. Note that Lemma 5.3 shows that $\phi_{D}\left(x^{-} 1_{\lambda}\right) \in \mathfrak{A}_{D}^{-}$for any $x \in \mathbf{f}$. Let $s_{D}: \mathbf{f} \rightarrow \mathfrak{A}_{D}^{-}$be given by

$$
x \mapsto \pi_{D}\left(\phi_{D}\left(x^{-} E_{i} 1_{\lambda}\right)\right)
$$

and define $r_{D}: \mathbf{f} \rightarrow \mathfrak{A}_{D}^{-}$by setting

$$
x \mapsto \frac{v^{-i \cdot \lambda}}{v-v^{-1}} \phi_{D}\left(r_{i}(x)^{-} 1_{\lambda}\right) .
$$

Corollary 5.6. Let $x \in \mathbf{f}$.

$$
s_{D}(x)-r_{D}(x)=\frac{v^{-2 p}}{v-v^{-1}}\left(\sum_{k=1}^{m} c_{k}(v)\left[{ }_{p} Z_{k}\right]\right)
$$

for some $Z_{k} \in \mathfrak{S}^{n, n}$ and $c_{k} \in \mathcal{A}$, independent of $p$. Hence the element $u(x)$ of (5-1) is orthogonal to $\mathbf{U}^{-} 1_{\lambda}$.

Proof. Let $y \in \mathbf{f}$ be a monomial. Then we have

$$
\left(u, y^{-} 1_{\lambda}\right)=\lim _{p \rightarrow \infty}\left\langle u, y^{-} 1_{\lambda}\right\rangle_{k+p n},
$$

and by definition

$$
\left\langle u, y^{-} 1_{\lambda}\right\rangle_{k+p n}=\left(s_{k+p n}(x)-r_{k+p n}(x), \phi_{k+p n}\left(y^{-} 1_{\lambda}\right)\right)_{k+p n} .
$$

By Lemma 5.4.

$$
s_{k+p n}(x)-r_{k+p n}(x)=v^{-2 p}\left(\sum_{j=1}^{m} c_{j}(v)\left[{ }_{p} Z_{j}\right]\right), \quad Z_{j} \in \mathfrak{S}^{n, n},
$$

and by Remark 4.4, we know that $\left(\left[{ }_{p} Z_{j}\right], \phi_{k+p n}\left(y^{-} 1_{\lambda}\right)\right)_{k+p n}$ converges in $\mathbb{Q}\left(\left(v^{-1}\right)\right)$ as $p \rightarrow \infty$. Thus the right-hand side of (5-4) tends to zero as required.

\section{Geometric interpretation}

Recall from [Lusztig $1999, \S 4$ ] that $\mathfrak{A}_{D}$ possesses a canonical basis $\mathfrak{B}_{D}$ consisting of elements $\{A\}, A \in \mathfrak{S}_{D, n, n}$. To define these elements we must assume $\mathbb{k}$ is algebraically closed (either the algebraic closure of $\mathbb{F}_{q}$, in which case we must use sheaves in the étale topology, or $\mathbb{C}$ in which case we use the analytic topology). Fix $A \in \mathfrak{S}_{D, n}$, and $\boldsymbol{L} \in \mathscr{F}_{r}(A)$.

The space $\mathscr{F}^{n}$ can be given the structure of an ind-scheme such that each set $X_{A}^{L}$ (see Section 3) lies naturally in a projective algebraic variety. This follows from the fact that if we fix $i_{0}, j_{0} \in \mathbb{Z}$, then the subsets

$$
\mathscr{F}_{\boldsymbol{b}, \boldsymbol{L}}^{p}=\left\{\boldsymbol{L}^{\prime} \in \mathscr{F}_{\boldsymbol{b}}: \varepsilon^{p} L_{i_{0}} \subset L_{j_{0}}^{\prime} \subset \varepsilon^{-p} L_{i_{0}}\right\}
$$


(for $p=1,2, \ldots$ ) are naturally projective algebraic varieties each embedded in the next, and for any fixed $A \in \mathfrak{S}_{D, n, n}$ there is a $p_{0} \in \mathbb{Z}$ such that $X_{A}^{L}$ is a locally closed subset of $\mathscr{F}_{b, L}^{p}$ for all $p \geq p_{0}$. Thus its closure $\bar{X}_{A}^{L}$ is naturally a projective algebraic variety. Let $\Phi_{A}^{L}$ (or sometimes for convenience just $\Phi_{A}$ ) denote the simple perverse sheaf on $\bar{X}_{A}^{L}$ whose restriction to $X_{A}^{L}$ is $\mathbb{C}\left[d_{A}\right]$. Let $\mathscr{H}^{s}\left(\Phi_{A}^{L}\right)$ be the $s$-th cohomology sheaf of $\Phi_{A}^{L}$. For $A_{1} \in \mathfrak{S}_{D, n, n}$ such that $X_{A_{1}}^{L} \subset \bar{X}_{A}^{L}$ we write $A_{1} \leq A$, and set

$$
\Pi_{A_{1}, A}=\sum_{s \in \mathbb{Z}} \operatorname{dim}\left(\mathscr{H}_{y}^{s-d_{A_{1}}}\left(\Phi_{A}^{\boldsymbol{L}}\right)\right) v^{s} \in \mathbb{Z}\left[v^{-1}\right],
$$

where $\mathscr{H}_{y}^{s-d_{A_{1}}}\left(\Phi_{A}^{\boldsymbol{L}}\right)$ is the stalk of $\mathscr{H}^{s-d_{A_{1}}\left(g_{A} \boldsymbol{L}\right)}$ at a point $y \in \bar{X}_{A_{1}}^{\boldsymbol{L}}$ (since $\mathscr{A}^{\boldsymbol{L}}$ is constructible with respect to the stratification of $\bar{X}_{A}^{L}$ given by $\left\{X_{A_{1}}^{L}: A_{1}<A\right\}$, this is independent of the choice of $y$ ). We let

$$
\{A\}=\sum_{A_{1} ; A_{1} \leq A} \Pi_{A_{1}, A}\left[A_{1}\right] .
$$

The next result is an immediate consequence of the definitions and Lemma 3.5. (It is the analogue for our inner product of [Lusztig 1999, Lemma 7.5]).

Lemma 6.1. Let $A, A^{\prime} \in \mathfrak{S}_{D, n, n}$. Then

$$
\left(\{A\},\left\{A^{\prime}\right\}\right)_{D} \in \delta_{A, A^{\prime}}+v^{-1} \mathbb{Z}\left[v^{-1}\right] .
$$

The algebra $\mathfrak{A}_{D}$ may be viewed as a convolution algebra of (equivariant) complexes on $\mathscr{F}^{n}$. One must be slightly careful here since one cannot (at least straightforwardly) consider convolution on $\mathscr{F}^{n} \times \mathscr{F}^{n}$ as the "complexes" one would then have to consider would have infinite-dimensional support. However, [Lusztig 1999, 4.2] gives one way in which this difficulty can be avoided: given $A, B \in \mathfrak{S}_{D, n, n}$ we may consider the set

$$
Z=\left\{\left(\boldsymbol{L}^{\prime}, \boldsymbol{L}^{\prime \prime}\right) \in \mathscr{F}_{\boldsymbol{b}} \times \mathscr{F}_{\boldsymbol{c}}: \boldsymbol{L}^{\prime} \in \bar{X}_{A}^{\boldsymbol{L}}, \boldsymbol{L}^{\prime \prime} \in \bar{X}_{B}^{\boldsymbol{L}^{\prime}}\right\} .
$$

As with $\bar{X}_{A}^{L}$ we see that $Z$ is naturally a projective variety and the projection $\pi$ to the second factor gives a proper map $Z \rightarrow \mathscr{F}_{c}$. The group $G_{L} \subset \operatorname{Aut}(V)$ of automorphisms stabilising $\boldsymbol{L}$ acts on $\mathscr{F}_{\boldsymbol{b}}$ through a quotient which is naturally an algebraic group, and thus it makes sense to speak of $G_{L}$-equivariant perverse sheaves on $\mathscr{F}_{\boldsymbol{b}}$ and $Z$. If $\mathscr{I}$ denotes the middle extension of the constant sheaf on the smooth locus of $Z$, then $\mathscr{I}$ has a canonical $G_{L}$-equivariant structure, and so by the decomposition theorem its push-forward along $\pi$ is a direct sum of (shifted) perverse sheaves of the form $\mathscr{I}_{C},\left(C \in \mathfrak{S}_{D, n, n}\right)$. We denote this push-forward by $\mathscr{I}_{A} * \mathscr{I}_{B}$. If $\mathscr{K}_{D, n, n}$ denotes the free $\mathscr{A}$-module on the set $\left[\mathscr{I}_{A}^{L}\right]$ of isomorphism classes of the sheaves $\Phi_{A}^{\boldsymbol{L}}$ (as $\boldsymbol{L}$ runs over a set of $\operatorname{Aut}(V)$-orbit representatives on $\mathscr{F}$ ) then the convolution $*$ gives $\mathscr{K}_{D, n, n}$ an associative $\mathscr{A}$-algebra structure, which 
is shown in [Lusztig 1999, 4.4] to be isomorphic to $\mathfrak{A}_{D, \mathscr{A}}$ via the map $\Theta$ given by $\Theta\left(\left[\mathscr{I}_{A}\right]\right)=\{A\}$. Moreover, Lusztig has shown that the submodule $K_{D, n, n}$ of $\mathscr{K}_{D, n, n}$ spanned by the elements $\left\{\left[\mathscr{I}_{A}\right]: A \in \mathfrak{S}_{D, n, n}^{\text {ap }}\right\}$ is precisely the preimage under $\Theta$ of the subalgebra $\mathbf{U}_{D, \mathscr{A}}$, where $\mathfrak{S}_{D, n, n}^{\text {ap }}$ is the subset of $\mathfrak{S}_{D, n, n}$ consisting of those matrices $A \in \mathfrak{S}_{D, n, n}$ for which, given any $p \in \mathbb{Z} \backslash\{0\}$, there is a $k \in \mathbb{Z}$ with $a_{k, k+p}=0$.

Remark 6.2. Note also that this isomorphism yields the existence of an $\mathscr{A}$-antilinear involution on $\mathfrak{A}_{D, \mathscr{A}}$ which fixes the basis elements $\{A\},\left(A \in \mathfrak{S}_{D, n, n}\right)$, by transporting via $\Theta$ the action of the Verdier duality functor. We will write this involution as $x \mapsto \bar{x}$. Since it fixes the generators $E_{i}(D), F_{i}(D), K_{a}$ it preserves the subalgebra $\mathbf{U}_{D, \mathscr{A}}$ and is compatible with the bar involution on $\dot{\mathbf{U}}$ (see [Lusztig 1999, 4.13] for more details). Moreover, Lusztig [Lusztig 1999, Proposition 4.12] shows that the antiautomorphism $\Psi$ corresponds to the map on $\mathscr{K}_{D, n, n}$ which sends $\left[\mathscr{I}_{A}\right]$ to $\left[\mathscr{I}_{A^{t}}\right]$.

We wish to give an interpretation of the inner product of Section 3 in the context of the algebra $\mathscr{K}_{D, n, n}$. Suppose that $A, B \in \mathfrak{S}_{D, n, n}$. We want to describe $(\{A\},\{B\})$. We may assume that $r(A)=r(B)=\boldsymbol{a}$ and $c(A)=c(B)=\boldsymbol{b}$. Let $\boldsymbol{L}^{\prime} \in \mathscr{F}_{\boldsymbol{b}}$. Let $\Phi_{A^{t}}^{\boldsymbol{L}^{\prime}}$ and $\Phi_{B^{t}}^{L^{\prime}}$ denote the simple perverse sheaves on $\bar{X}_{A^{t}}^{L^{\prime}}$ and $\bar{X}_{B^{t}}^{L^{\prime}}$ respectively. Then define

$$
\left\langle\Phi_{A}, \Phi_{B}\right\rangle^{D}=\sum_{i \in \mathbb{Z}} \operatorname{dim}\left(H_{c}^{i}\left(\mathscr{F}_{\boldsymbol{a}}, \Phi_{A^{t}}^{\boldsymbol{L}^{\prime}} \otimes \mathscr{I}_{B^{t}}^{\boldsymbol{L}^{\prime}}\right)\right) v^{i} .
$$

(Here as usual $\otimes$ denotes the derived tensor product.) Clearly $\langle\cdot, \cdot\rangle^{D}$ extends to an inner product on the whole of $\mathfrak{A}_{D}$ (viewed as an algebra of equivariant complexes on $\left.\mathscr{F}^{n}\right)$. We want to show that it is the same as the inner product $(\cdot, \cdot)_{D}$ of Section 3 , at least on the subalgebra $\mathbf{U}_{D}$. We start by showing that $\langle\cdot, \cdot\rangle^{D}$ satisfies the properties of Corollary 3.3.

Lemma 6.3. Let $A, B, C \in \mathfrak{S}_{D, n, n}$, and suppose that $\mathbb{O}_{A}$ is a closed orbit. Then

$$
\left\langle\mathscr{I}_{A} * \mathscr{I}_{B}, \mathscr{I}_{C}\right\rangle^{D}=v^{d_{A}-d_{A^{t}}}\left\langle\mathscr{I}_{B}, \mathscr{I}_{\left.A^{t} * \mathscr{I}_{C}\right\rangle^{D} .} .\right.
$$

Proof. Both sides are obviously zero unless $r(A)=r(C)=\boldsymbol{a}, c(A)=r(B)=\boldsymbol{b}$ and $c(B)=c(C)=\boldsymbol{c}$; thus we assume these equalities from now on. Pick $\boldsymbol{L}^{0} \in \mathscr{F}_{\boldsymbol{c}}$, and pick a subset $Y=\mathscr{F}_{b, L^{0}}^{p}$ of $\mathscr{F}_{\boldsymbol{b}}$ large enough that $Y$ is a smooth projective variety containing $X_{B^{t}}^{L_{0}}$. Let

$$
Z_{A}=\left\{\left(\boldsymbol{L}, \boldsymbol{L}^{\prime}\right) \in \mathbb{O}_{A}: \boldsymbol{L}^{\prime} \in Y\right\},
$$

We have maps $p_{1}: Z_{A} \rightarrow \mathscr{F}_{a}$ and $p_{2}: Z \rightarrow Y$, the first and second projections respectively. The map $p_{1}$ is clearly proper (as the fibre is $X_{A}^{L} \cap Y$ ) and the map $p_{2}$ is smooth with fibre dimension $d_{A^{t}}$. It follows that the complex $\mathscr{I}$ used in the definition of $\mathscr{I}_{A} * \mathscr{I}_{B}$ is the pull-back $p_{2}^{*}\left(\mathscr{I}_{B^{t}}\right)\left[d_{A^{t}}\right]$, and hence we have

$$
\left(\Phi_{A} * \Phi_{B}\right)^{t}=\left(p_{1}\right) ! p_{2}^{*}\left(\Phi_{B^{t}}\right)\left[d_{A^{t}}\right],
$$


and thus in particular

$$
\begin{aligned}
\left(\mathscr{I}_{A} * \mathscr{I}_{B}\right)^{t} \otimes \mathscr{I}_{C^{t}} & =\left(p_{1}\right) ! p_{2}^{*}\left(\mathscr{I}_{B^{t}}\right)\left[d_{A^{t}}\right] \otimes \mathscr{I}_{C^{t}} \\
& =\left(p_{1}\right) !\left(p_{2}^{*}\left(\mathscr{I}_{B^{t}}\right) \otimes p_{1}^{*}\left(\mathscr{I}_{C^{t}}\right)\left[d_{A^{t}}\right]\right),
\end{aligned}
$$

where we use the projection formula in the second equality.

On the other hand, to compute the product $\mathscr{I}_{A^{t}} * \mathscr{I}_{C}$ we may similarly pick a smooth projective variety $W \subset \mathscr{F}_{\boldsymbol{a}}$ which contains $X_{C^{t}}^{\boldsymbol{L}^{0}}$, and consider the variety

$$
Z_{A^{t}}=\left\{\left(\boldsymbol{L}, \boldsymbol{L}^{\prime}\right) \in \mathcal{O}_{A}: \boldsymbol{L} \in W\right\} .
$$

As above there are projection maps $p_{1}, p_{2}$, and the product is given by

$$
\left(\Phi_{A^{t} *} \Phi_{C}\right)^{t}=\left(p_{2}\right) ! p_{1}^{*}\left(\Phi_{C^{t}}\right)\left[d_{A}\right] .
$$

so that

$$
\begin{aligned}
\mathscr{I}_{B^{t}} \otimes\left(\mathscr{I}_{\left.A^{t} * \mathscr{I}_{C}\right)^{t}}\right. & =\mathscr{I}_{B^{t}} \otimes\left(p_{2}\right) ! p_{1}^{*}\left(\mathscr{I}_{C^{t}}\right)\left[d_{A^{t}}\right] \\
& =\left(p_{2}\right) !\left(p_{2}^{*}\left(\mathscr{I}_{B^{t}}\right) \otimes p_{1}^{*}\left(\mathscr{I}_{C^{t}}\right)\right)\left[d_{A}\right]
\end{aligned}
$$

where we again use the projection formula. Now since tensor product is local, we may restrict to $Z_{A} \cap Z_{A^{t}}$, and then it is clear that both inner products are given by the compactly supported cohomologies of $p_{2}^{*}\left(\mathscr{I}_{B^{t}}\right) \otimes p_{1}^{*}\left(\mathscr{I}_{C^{t}}\right)$ up to shift, with the difference in shifts being $d_{A}-d_{A^{t}}$ as required.

Lemma 6.4. Let $A, B \in \mathfrak{S}_{D, n, n}$, and $\boldsymbol{c} \in \mathfrak{S}^{n}$. Then

(1) $\left\langle E_{i}\{A\},\{B\}\right\rangle^{D}=\left\langle\{A\}, v K_{i} F_{i}\{B\}\right\rangle^{D}$,

(2) $\left\langle F_{i}\{A\},\{B\}\right\rangle^{D}=\left\langle\{A\}, v K_{-i} E_{i}\{B\}\right\rangle^{D}$,

(3) $\left\langle K_{c}\{A\},\{B\}\right\rangle^{D}=\left\langle\{A\}, K_{c}\{B\}\right\rangle^{D}$.

Proof. This follows from the previous lemma exactly as in the proof of Corollary 3.3, since the varieties $X_{a+i}^{L} e_{a}$ are closed.

The algebra $\mathbf{U}_{D}$ is spanned by elements of the form $T_{1} T_{2} \ldots T_{N}\left[\boldsymbol{i}_{\boldsymbol{a}}\right]$ where $T_{s}$ is either $E_{i}$ or $F_{i}$ for some $i$. Thus, by Corollary 3.3, in order to show that the inner products ()$_{D}$ and $\langle\cdot, \cdot\rangle^{D}$ coincide via the isomorphism the previous lemma shows we need only check that

$$
\left\langle T_{1} T_{2} \ldots T_{N}\left[\boldsymbol{i}_{\boldsymbol{a}}\right],\left[\boldsymbol{i}_{\boldsymbol{a}}\right]\right\rangle^{D}=\left(T_{1} T_{2} \ldots T_{N}\left[\boldsymbol{i}_{\boldsymbol{a}}\right],\left[\boldsymbol{i}_{\boldsymbol{a}}\right]\right)_{D}
$$

But this will follow if we can show that

$$
\left\langle\{A\},\left[\boldsymbol{i}_{\boldsymbol{a}}\right]\right\rangle^{D}=\left(\{A\},\left[\boldsymbol{i}_{\boldsymbol{a}}\right]\right)_{D}
$$

for all $A \in \mathfrak{S}_{D, n, n}$, as $\left\{\{A\}: A \in \mathfrak{S}_{D, n, n}\right\}$ is a basis of $\mathfrak{A}_{D}$. The simple perverse sheaf corresponding to $\left\{\boldsymbol{i}_{\boldsymbol{a}}\right\}=\left[\boldsymbol{i}_{\boldsymbol{a}}\right]$ is just the skyscraper sheaf at the point $\boldsymbol{L}^{\prime}$; hence this last equality follows from directly from the definitions. We have therefore shown the following result. 
Proposition 6.5. On the algebra $\mathbf{U}_{D}$ the inner products $\langle\cdot, \cdot\rangle^{D}$ and $(\cdot, \cdot)_{D}$ coincide.

Remark 6.6. It can be shown that the algebra $\mathfrak{A}_{D}$ is generated by the elements $\{A\}$ for which $X_{A}^{L}$ is closed, and so the above argument adapts to show that the inner products in fact agree on the whole of $\mathfrak{A}_{D}$, but we will not need this. Henceforth we will use the notation $(\cdot, \cdot)_{D}$ when referring to the inner product on $\mathfrak{A}_{D}$ in either of its incarnations.

We now give a second proof of the agreement of the limit of the inner products on $\mathbf{U}_{D}$ with Lusztig's inner product on $\dot{\mathbf{U}}$. Recall that Theorem 5.1 characterises the inner product by three properties, the first two of which are evident for our limiting inner product. The difficulty then is establishing the third property, which relates the inner product on $\dot{\mathbf{U}}$ to that on $\mathbf{f}$. We give a proof of this property, which while less elementary than the proof in Section 5 is more conceptual. In the remainder of this section we will assume that our base field $\mathbb{k}$ is the field of complex numbers $\mathbb{C}$, and work with sheaves in the analytic topology. We thus briefly review some basics of the equivariant derived category, following the approach of [Bernstein and Lunts 1994].

We first need to recall the geometric construction of the algebra $\mathbf{f}$ and its inner product (at least in the case of the cyclic quiver). Let $Q$ be the cyclic quiver $1 \rightarrow 2 \rightarrow \cdots \rightarrow n \rightarrow 1$. A representation of $Q$ is a $\mathbb{Z} / n \mathbb{Z}$-graded vector space $W=\bigoplus_{i \in \mathbb{Z} / n \mathbb{Z}} W_{i}$ equipped with linear maps $y_{i}: W_{i} \rightarrow W_{i+1}$ (where $i \in \mathbb{Z} / n \mathbb{Z}$ ). The space of such representations is denoted $E_{W}$. Such a representation is nilpotent if there is an $N>0$ such that all compositions of $y_{i}$ s of length greater than $N$ are equal to zero, and we write $E_{W}^{\text {nil }}$ for the subvariety of nilpotent representations. The group $G_{W}=\prod_{i \in \mathbb{Z} / n \mathbb{Z}} \mathrm{GL}\left(V_{i}\right)$ acts on $E_{W}^{\text {nil }}$ with finitely many orbits. The algebra f associated to the Cartan datum of affine type $\widehat{\mathfrak{s l}}_{n}$ is then given as a convolution algebra of semisimple perverse sheaves which are $G_{W}$-equivariant and are supported on $E_{V}^{\text {nil }}$; thus, since the stabiliser of a nilpotent representation is connected, the simple objects are labelled by the $G_{W}$-orbits on $E_{W}^{\text {nil }}$, that is, by the isomorphism classes of nilpotent representations.

Given a pair $(t, m) \in \mathbb{Z} \times \mathbb{Z}_{\geq 0}$ we have a representation $V_{t, m}$ of the cyclic quiver with basis $\left\{e_{j}: t \leq j \leq t+p-1\right\}$ where $e_{j}$ has degree $j \bmod n$, and $e_{t} \rightarrow e_{t+1} \rightarrow$ $\cdots e_{t+p-1} \rightarrow 0$. The representations $V_{t, p}$ are a complete set of representatives for the isomorphism classes of indecomposable nilpotent representations of the cyclic quiver; thus, since any nilpotent representation is a direct sum of indecomposables, we can record the isomorphism class of any such representation by a tableau $\left(\mu_{t, p}\right)_{t, p \in \mathbb{Z}}$ where the entry $\mu_{t, p}$ records the multiplicity of $V_{t, m}$ in the representation. We therefore have a natural parametrisation of the canonical basis $\mathbf{B}$ by tableaux

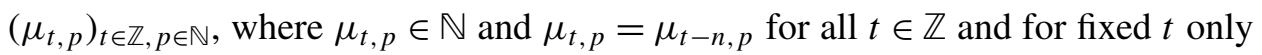
finitely many of the $\mu_{t . p}$ are nonzero. 
If $\operatorname{dim}\left(W_{i}\right)=v_{i},(i \in \mathbb{Z} / n \mathbb{Z})$, the orbits of $G_{W}$ on $E_{W}^{\text {nil }}$ correspond to the isomorphism classes of nilpotent representations of dimension $v$ hence they are labelled by the set $\Sigma_{v}$ consisting of those tableaux $\left(\mu_{t, p}\right)$ for which

$$
\sum_{\substack{t, p \\ t \leq k<t+p}} \mu_{t, p}=v_{k},
$$

and so this same set indexes the $v$-homogeneous part of $\mathbf{f}$.

The inner product on $\mathbf{f}$ is defined in [Lusztig 1993, §12.2]. Let $A_{1}, A_{2}$ be $G_{W^{-}}$ equivariant simple perverse sheaves on $E_{W}^{\text {nil }}$. Note that the definition there is simply an explicit calculation of

$$
\left(A_{1}, A_{2}\right)=\sum_{j \in \mathbb{Z}} \operatorname{dim}\left(H_{G, c}^{j}\left(A_{1} \otimes A_{2}\right)\right) v^{-j} .
$$

where $H_{G, c}^{*}$ denotes equivariant cohomology with compact supports (see below for more details).

Definition 6.7. Let $X$ be a variety with a $G$-action (or more compactly, a $G$-variety). A resolution of $X$ is a map $p: P \rightarrow X$ where $P$ is smooth $G$-variety on which $G$ acts freely (so that $\bar{P}=P / G$ is a smooth variety also). Let $\pi: P \rightarrow \bar{P}$ denote the quotient map. The category $D_{G}^{b}(X, P)$ consists of triples $(\mathscr{F}, \mathscr{G}, \phi)$ where $\mathscr{F}$ is an object in $D^{b}(\bar{P})$ and $\mathscr{G}$ is an object in $D^{b}(X)$ and $\phi: \pi^{*}(\mathscr{F}) \rightarrow p^{*}(\mathscr{G})$ is an isomorphism.

We will also need to recall the notion of an $n$-acyclic map.

Definition 6.8. A map $f: Y \rightarrow X$ is said to be $n$-acyclic if it has the following properties:

(i) For any sheaf $F$ on $Y$ the adjunction morphism $B \rightarrow R^{0} f_{*} f^{*}(B)$ is an isomorphism, and $R^{i} f_{*} f^{*}(F)=0$ for $0<i \leq n$.

(ii) For any base change $\tilde{X} \rightarrow X$ the induced map $\tilde{f}: \tilde{Y}=Y \times_{X} \tilde{X} \rightarrow \tilde{X}$ has property (i).

If we write $\tau_{\leq n}$ for the truncation functor on the derived category $D^{b}(Y)$, the first condition may be rewritten as saying that the adjunction map $F \rightarrow \tau_{\leq n} R f_{*} f^{*}(F)$ in $D^{b}(Y)$ is an isomorphism for any sheaf $F$ (thought of as an complex in $D^{b}(Y)$ concentrated in degree 0$)$.

For sufficiently acyclic resolutions $P$ (i.e., resolutions $p: P \rightarrow X$ with $p$ an $n$-acyclic map for $n$ large), the cohomologies of objects in the category $D_{G}^{b}(X, P)$ can be used to calculate the cohomologies in $D_{G}^{b}(X)$ as indeed Bernstein and Lunts take a limit of resolution of $X$ to obtain their definition of the equivariant derived category. The construction in [Lusztig 1993, §12.2] gives an explicit construction 
of a collection of $G$-resolutions of a variety which can be made arbitrarily highly connected (and hence his definition is the same as that of (6-3)) however for our comparison result we need a more flexible context.

To compare the inner products on $\mathbf{f}$ and $\mathbf{U}_{D}$ we need to relate the geometry of periodic lattices to the cyclic quiver. The description of the relation we need goes back to [Lusztig 1990, §11], and is also used in [Ginzburg and Vasserot 1993]. Here we follow the presentation of [Lusztig 1999]. Suppose that $\boldsymbol{L}$ is a fixed lattice, and $\boldsymbol{a} \in \mathfrak{S}^{n}$ is such that $\operatorname{dim}\left(V_{i}\right)=a_{i}$ (where on the left-hand side of this equality $i$ is understood to be taken modulo $n$ ). Consider the following spaces:

- $\mathscr{X}_{\boldsymbol{a}, \nu}^{\boldsymbol{L}}=\left\{\boldsymbol{L}^{\prime} \in \mathscr{F}_{\boldsymbol{a}}: L_{i}^{\prime} \subseteq L_{i}\right.$ and $\operatorname{dim}\left(L_{i} / L_{i}^{\prime}\right)=v_{i}$ for all $\left.i \in \mathbb{Z}\right\}$.

- $\tilde{\mathscr{X}}_{\boldsymbol{a}, v}^{\boldsymbol{L}}=\left\{\left(\boldsymbol{L}^{\prime},\left(\phi_{i}\right)_{i \in \mathbb{Z}}: \boldsymbol{L}^{\prime} \in \mathscr{X}_{\boldsymbol{a}, v}^{\boldsymbol{L}}\right.\right.$ and $\phi_{i}: L_{i} / L_{i}^{\prime} \rightarrow V_{i}$ is an isomorphism $\}$.

- $u_{\boldsymbol{a}} \subset E_{W}^{\mathrm{nil}}$ consists of those representations with label $\left(\mu_{t, p}\right)$ such that

$$
\mu_{t, 1}+\mu_{t, 2}+\cdots \leq a_{t} \quad \text { for all } t \in \mathbb{Z} .
$$

Both $\mathscr{X}_{\boldsymbol{a}, v}^{\boldsymbol{L}}$ and $\tilde{\mathscr{X}}_{\boldsymbol{a}, v}^{\boldsymbol{L}}$ can be given a natural structure of algebraic variety (with $\mathscr{L}_{\boldsymbol{a}, v}^{\boldsymbol{L}}$ projective), in the same fashion as for $\bar{X}_{\boldsymbol{L}}^{A}$ above, and the variety $\boldsymbol{U}_{\boldsymbol{a}}$ is an open subset of $E_{W}^{\text {nil }}$ (see [Lusztig 1999, Lemma 5.8]). We then have the correspondence

$$
\mathscr{X}_{\boldsymbol{a}, v}^{\boldsymbol{L}} \stackrel{\alpha}{\alpha}^{\boldsymbol{L}} \stackrel{\beta}{\longrightarrow} \mathscr{\mathscr { L }}_{\boldsymbol{a}, v},
$$

where the map $\alpha$ is given by $(\boldsymbol{L}, \phi) \mapsto \boldsymbol{L}$, while the map $\beta$ is given by sending $(\boldsymbol{L}, \phi)$ to the element $\left(y_{i}\right) \in E_{V}^{\text {nil }}$ where $y_{i}$ given by the composition

$$
V_{i} \stackrel{\phi_{i}^{-1}}{\longrightarrow} L_{i} / L_{i}^{\prime} \longrightarrow L_{i+1} / L_{i+1}^{\prime} \stackrel{\phi_{i+1}}{\longrightarrow} V_{i+1},
$$

with the middle map induced by the inclusion $L_{i} \subseteq L_{i+1}$ (the point $\left(y_{i}\right)_{i \in \mathbb{Z} / n \mathbb{Z}}$ is automatically nilpotent as a representation of $Q$ by the periodicity of the flags $\left.\boldsymbol{L}, \boldsymbol{L}^{\prime}\right)$. The map $\alpha$ is clearly a principal $G_{W}$-bundle, while the map $\beta$ is smooth with connected fibres of dimension $\sum_{1 \leq i \leq n} a_{i} v_{i}$ (see [Lusztig 1999, Lemma 5.11]).

Notice that if $\boldsymbol{a}$ has $a_{i}$ large enough for all $i$, then we have $E_{W}^{\text {nil }}=u_{\boldsymbol{a}}$. In what follows we will always assume that this is the case. Moreover, the groups $G_{\boldsymbol{L}}$ (that is, the group of automorphisms of $V$ which preserve the lattice $L$ ) and $G_{W}$ act naturally on $\tilde{\mathscr{X}}_{\boldsymbol{a}, \boldsymbol{b}}^{\boldsymbol{L}}$, making the maps $\alpha$ and $\beta$ equivariant (for the actions of $G_{\boldsymbol{L}}$ on $\mathscr{X}_{\boldsymbol{a}, \boldsymbol{L}}$ and $G_{W}$ on $E_{W}^{\mathrm{nil}}$ ). Thus since $\tilde{\mathscr{X}}_{\boldsymbol{a}, \nu}^{\boldsymbol{L}}$ is free $G_{W}$-space (using the map $\alpha$ ) it is a resolution of $E_{W}^{\text {nil }}$.

Now Lusztig has shown in [Lusztig 1999, §5] that if $b$ is an element of the canonical basis which corresponds to the simple perverse sheaf $P$ on $E_{W}^{\text {nil }}$ with associated $G_{W}$-orbit corresponding to the tableau $\left(\mu_{t, p}\right)$ then $\phi_{D}(b)\left[\boldsymbol{i}_{\boldsymbol{a}}\right] \in \mathfrak{A}_{D, n, n}$ is the element $\{B\}$ where $b_{i, i+j}=\mu_{i, j}$ and $b_{i i}=a_{i}-\sum_{p>0} \mu_{i, p}$, and $b_{i j}=0$ if 
$i>j$. Moreover, we have

$$
\alpha^{*}\left(\Phi_{B}\right) \cong \beta^{*}(P)
$$

Picking an isomorphism $\theta$ (which is unique up to a scalar since $A$ is simple) we therefore obtain an element $\tilde{P}=\left(\alpha^{*}\left(\mathscr{I}_{B}\right), P, \theta\right)$ of $D_{G}^{b}\left(E_{W}^{\text {nil }}, \tilde{\mathscr{X}}_{\boldsymbol{a}, \nu}^{\boldsymbol{L}}\right)$. Thus if $b_{1}, b_{2}$ are elements of $\mathbf{B}_{v}$ with associated tableau $\left(\mu_{t, p}\right)$ and $\left(\rho_{t, p}\right)$, and $P_{1}, P_{2}$ the corresponding perverse sheaves on $E_{W}^{\text {nil }}$, and $B_{1}$ and $B_{2}$ are the associated elements of $\mathfrak{S}_{D, n, n}^{-}$then we may choose elements $\tilde{P}_{k}=\left(\Phi_{B_{k}}, P_{k}, \theta_{k}\right)$ (where $\left.k=1,2\right)$ in the category $D_{G}^{b}\left(E_{W}^{\mathrm{nil}}, \tilde{\mathscr{X}}_{\boldsymbol{a}, v}^{\boldsymbol{L}}\right)$ such that

$$
\left(\Phi_{A}, \Phi_{B}\right)_{D}=\sum_{j \in \mathbb{Z}} H_{c}^{j}\left(\tilde{P}_{1} \otimes \tilde{P}_{2}\right) v^{-j},
$$

where $H_{c}^{j}\left(\tilde{P}_{1} \otimes \tilde{P}_{2}\right)$ denotes the cohomology with compact supports of the object $\tilde{P}_{1} \otimes \tilde{P}_{2}$ in the category $D_{G}^{b}\left(E_{W}^{\text {nil }}, \tilde{\mathscr{C}}_{\boldsymbol{a}, v}^{\boldsymbol{L}}\right)$. It follows that if we consider $\boldsymbol{a}^{\prime}=\boldsymbol{a}+p \boldsymbol{b}_{0}$ instead of $\boldsymbol{a}$ for larger and larger $p$, this inner product will converge to $\left(b_{1}, b_{2}\right)$ provided the resolutions $\tilde{\mathscr{x}}_{\boldsymbol{a}^{\prime}, v}$ become more and more highly connected as the $a_{i}^{\prime} \rightarrow \infty$. Thus the compatibility of the inner products is reduced to showing that the maps $\beta: \tilde{X}_{\boldsymbol{a}, \nu}^{L} \rightarrow E_{W}^{\text {nil }}$ is $k$-connected where $k \rightarrow \infty$ as $\min \left\{a_{i}\right\} \rightarrow \infty$. The rest of this section will be devoted to a proof of this result.

We wish to use a general lemma which gives a criterion for a map to be $n$-acyclic. Since we cannot find a precise reference for what we need, we sketch the result, though it is presumably well-known to the experts. The statement is a version of the Vietoris-Begle theorem proved in [Kashiwara and Schapira 1994, Proposition 2.7.8].

Lemma 6.9. Suppose we have a map $f: Y \rightarrow X$ which has $k$-connected fibres, and that we may exhaust $Y=\bigcup_{n} Y_{n}$, by closed subsets $Y_{n}$ such that $Y_{n} \subset \operatorname{Int}\left(Y_{n+1}\right)$ and the restriction of $f$ to $Y_{n}$ is proper with k-connected fibres for all $n$, then $\tau_{\leq k} R f_{*} \circ f^{*} \cong \mathrm{id}$.

Proof. In fact the reference [Kashiwara and Schapira 1994, Proposition 2.7.8] more is proved under the assumption that the fibres of $f$ are contractible, but the weaker statement that we need is precisely what follows from the proof given there. The key point is that in the case where $f$ is proper, one may use proper base change to conclude the vanishing of the functors $R f_{*}^{j} f^{*}$ in the appropriate range from the $k$-connectedness of the fibres. The extension to the noncompact case then follows via the Mittag-Leffler condition.

Since the hypotheses of Lemma 6.9 are preserved by base change, it yields a criterion for a map to be $n$-acyclic. We now use the above lemma to show that $\beta$ is a $k$-acyclic map for $k=\min _{0 \leq i \leq n-1}\left\{\left(a_{i}-v_{i}\right)\right\}$.

Lemma 6.10. The fibres of $\beta: \tilde{\mathscr{X}}_{\boldsymbol{a}, v}^{\boldsymbol{L}} \rightarrow \mathcal{U}_{\boldsymbol{a}}$ are $k$-connected for $k=2 \min _{1 \leq i \leq n}\left\{\left(a_{i}-v_{i}\right)\right\}$. 
$p t$

Proof. First note that we may view $X=\tilde{\mathscr{X}}_{\boldsymbol{a}, \nu}^{\boldsymbol{L}}$ as the set

$$
\left\{\left(\varphi_{i}\right)_{i \in \mathbb{Z}}: \varphi_{i}: L_{i} / \operatorname{ker}\left(\varphi_{i-1}\right) \rightarrow W_{i}\right\},
$$

where $\varphi_{i}$ is surjective, $\operatorname{ker}\left(\varphi_{i}\right)$ is a lattice, and $\varphi_{i-n}=\epsilon \varphi_{i} \epsilon^{-1}$. The corresponding pair $\left(\boldsymbol{L}^{\prime},\left(\phi_{i}\right)\right)$ is given by $\boldsymbol{L}^{\prime}=\left(\operatorname{ker}\left(\varphi_{i}\right)\right)_{i \in \mathbb{Z}}$ with the isomorphisms $\phi_{i}: L_{i} / L_{i}^{\prime} \rightarrow W_{i}$ induced by the surjections $\varphi_{i}$.

Now suppose that $y=\left(y_{i}\right)_{i \in \mathbb{Z} / n \mathbb{Z}} \in E_{W}^{\text {nil }}$ is a nilpotent representation of the cyclic quiver, and that $\left(\varphi_{i}\right)_{i \in \mathbb{Z}}$ is in the fibre of $y$. Considering the diagram

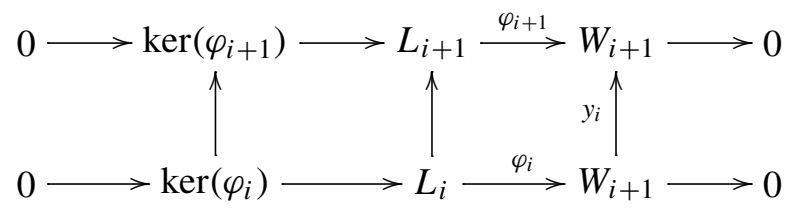

we see that the restriction of $\varphi_{i+1}$ is determined on $L_{i}$ as it is given by $y_{i} \circ \varphi_{i}$ there. Thus given $\varphi_{i}$, the collection of $\varphi_{i+1} \mathrm{~s}$ which induce $y_{i}$ is given by choosing a surjection $\pi: L_{i+1} / L_{i} \rightarrow W_{i+1} / \operatorname{im}\left(y_{i}\right)$, and then picking a lift of the pair of maps $\left(\pi, y_{i} \circ \varphi_{i}\right)$ to a map $\varphi_{i+1}: L_{i} / \operatorname{ker}\left(\varphi_{i}\right) \rightarrow W_{i+1}$ (since any such lift will be a surjective map). Thus the space of such choices is homotopy equivalent to the space of surjections from $L_{i+1} / L_{i}$ to $W_{i+1} / \operatorname{im}\left(y_{i}\right)$. This is a complex Stiefel manifold, and hence $2\left(a_{i}-v_{i}+\operatorname{rank}\left(y_{i}\right)\right)$ connected.

Thus if we set $k=2 \min _{1 \leq i \leq n}\left\{\left(a_{i}-v_{i}\right)\right\}$ we may view $\beta^{-1}(y)$ as an iterated sequence of fibre bundles over the space $B$ of surjections $\left\{\varphi_{0}: L_{0} \rightarrow W_{0}\right\}$ where in each case the fibres are at least $k$-connected. Thus by the standard long exact sequence $\beta^{-1}(y)$ will be $k$-connected provided we can show that $B$ is. Now $B$ is the space of surjective linear maps from $L_{0}$ to $W_{0}$ which intertwine the action of $\epsilon$ with the composition $\theta=y_{n} y_{n-1} y_{n-2} \ldots y_{0}: W_{0} \rightarrow W_{0}$ (note that since the action of $\epsilon$ is nilpotent, this shows the representation $y$ must be also) and the following lemma shows that in fact $B$ is at least $2\left(D-v_{0}\right) \geq 2\left(a_{0}-v_{0}\right)$ connected, so we are done.

Lemma 6.11. Let $(U, \theta)$ be a finite-dimensional vector space $U$ equipped with nilpotent endomorphism $\theta$ of Jordan type $\lambda$, and let $L$ be a free $\mathbb{C}[\epsilon]$-module of rank $D$. Viewing $U$ as $a \mathbb{C}[\epsilon]$-module via $\epsilon \mapsto \theta$, the space of $\mathbb{C}[\epsilon]$-module surjections $\varphi: L \rightarrow U$ is $d$-connected, where $d=2(D-\ell(\lambda))$ and $\ell(\lambda)$ is the length of the partition $\lambda$.

Proof. A $k[\epsilon]$-module map $\varphi: L \rightarrow U$ is surjective if and only if the induced k-linear map $\bar{\varphi}: L / \epsilon(L) \rightarrow U / \theta(U)$ is surjective. Moreover, since the space of surjections from $L / \epsilon(L)$ to $U / \theta(U)$ is a complex Stiefel manifold of $k$-frames in a $D$ dimensional space, it is $2(D-\ell(\lambda))$-connected. 
Now suppose that $\psi$ is a $\mathbb{C}$-linear map from $L / \epsilon(L) \rightarrow U / \theta(U)$. Let $K$ denotes the $D$-dimensional $\mathbb{C}$-vector space spanned by a set of $\mathbb{C}[\epsilon]$-generators of $L$, say $\left\{e_{1}, \ldots, e_{D}\right\}$. A $k[\epsilon]$-module map $\varphi: L \rightarrow U$ with $\bar{\varphi}=\psi$ is completely determined by its restriction to $K$, and the induced map $\varphi^{\prime}: K \rightarrow U$ is given by a choice of lifts for the vectors $\left\{\psi\left(e_{i}\right)\right\}$ in $U$ (where by abuse of notation we denote by $\psi$ the composition of $L \rightarrow L / \epsilon(L) \rightarrow U / \theta(U))$, and hence the space of such surjections is clearly a vector bundle over the Stiefel manifold of surjections from $L / \epsilon L$ to $U / \theta(U)$ which proves the lemma.

Proposition 6.12. The map $\beta: \tilde{\mathscr{X}}_{\boldsymbol{a}, v}^{\boldsymbol{L}} \rightarrow E_{W}^{\text {nil }}$ is $k$-connected.

Proof. To show $\beta$ is a $k$-connected map we wish to apply Lemma 6.9. By Lemma 6.10 we know that the fibres of $\beta$ are $k$-connected, and hence we must show that we can filter $\tilde{\mathscr{X}}_{\boldsymbol{a}, v}^{\boldsymbol{L}}$ by subvarieties $\left\{Y_{i}\right\}_{i \in \mathbb{N}}$ such that $\beta_{\mid Y_{i}}$ is proper while ensuring that the fibres remain $k$-connected. To do this we simply note that the topology of the fibres of $\beta$ all come from Stiefel manifolds, and these deformation retract on to the compact Stiefel manifolds. Moreover the retraction can be done via the Gram-Schmidt process, once we endow our vector spaces with a Hermitian inner product.

More precisely, we may equip $V$ with an Hermitian inner product (here we will assume that $\mathrm{k}=\mathbb{C}$, as in the rest of this section) so that $\epsilon$ is a unitary map (e.g., take a $\mathbb{C}[\epsilon]$-basis $\left\{e_{1}, e_{2}, \ldots e_{D}\right\}$ of $L_{0}$ and define the Hermitian product $\langle\cdot, \cdot\rangle^{D}$ by setting

$$
\left\langle\epsilon^{l} e_{j}, \epsilon^{m} e_{k}\right\rangle=\delta_{j, k} \delta_{l, m}, \quad(l, m \in \mathbb{Z}, 1 \leq j, k \leq D),
$$

Similarly we may equip the $\left\{W_{i}\right\}$ with Hermitian inner products. Then we have a norm function $\mathrm{N}$ on $\tilde{\mathscr{X}}_{\boldsymbol{a}, \boldsymbol{L}}^{\boldsymbol{L}}$ given by

$$
\mathrm{N}\left(\boldsymbol{L},\left(\phi_{i}\right)\right)=\max _{0 \leq i \leq n-1}\left\{\sup \left\{\left\|\phi_{i}(u)\right\|: u \in L_{i} / L_{i}^{\prime},\|u\|=1\right\} .\right.
$$

where the norm on $L_{i} / L_{i}^{\prime}$ is induced from that on $V$ via the canonical isomorphism $\left(L_{i}^{\prime}\right)^{\perp} \cong L_{i} / L_{i}^{\prime}$. Now we may set $Y_{i}=\left\{\left(\boldsymbol{L},\left(\phi_{i}\right)\right): i^{-1} \leq \mathrm{N}\left(\boldsymbol{L},\left(\phi_{i}\right)\right) \leq i\right\}$. Since the map $\alpha$ from $\tilde{\mathscr{X}}_{\boldsymbol{a}, v}^{\boldsymbol{L}}$ to $\mathscr{X}_{\boldsymbol{a}, v}^{\boldsymbol{L}}$ is a principal $G_{W}$-bundle over a projective variety, and the norm condition defining $Y_{i}$ clearly cuts out a compact subset of the fibres of $\alpha$, it follows that $Y_{i}$ is compact, and so in particular $\beta_{\mid Y_{i}}$ is proper. Thus it remains to check that the fibres of $\beta_{\mid Y_{i}}$ are still $k$-connected.

To do this we may use the Gram-Schmidt process to iteratively deform the linear maps in the fibres in the same manner as we checked $k$-connectedness via a sequence of fibre bundles. In the case of the choice of the surjection $L_{0} \rightarrow W_{0}$ which defines $L_{0}^{\prime}$ and $\phi_{0}$, note that we need only apply Gram-Schmidt to the frame defining the map from $K$ to $W_{0} / \theta\left(W_{0}\right)$, where $K=\operatorname{span}_{\mathbb{C}}\left\{e_{1}, \ldots, e_{D}\right\}$.

We can now complete the geometric proof of the equality of our inner product with that in [Lusztig 1993]. 
Theorem 6.13. The inner products $\langle\cdot, \cdot\rangle$ and $(\cdot, \cdot)$ on $\dot{\mathbf{U}}$ coincide.

Proof. As discussed above, it is enough to know that the varieties $\tilde{\mathscr{X}}_{\boldsymbol{a}+p \boldsymbol{b}_{0}, v}^{\boldsymbol{L}}$ become more and more connected as $p$ tends to infinity. Since the value of $k=\min _{0 \leq i \leq n-1}\left\{\left(a_{i}+p-v_{i}\right)\right\}$ clearly tends to infinity as $p$ does (where $\boldsymbol{a}$ is the representative of $\lambda \in X^{\prime}$ lying in $\left.\mathfrak{S}_{D, n}\right)$ the equality of the inner products follows.

\section{A construction of the canonical basis of $\dot{\mathrm{U}}\left(\widehat{\mathfrak{s l}}_{n}\right)$}

Lusztig [2000] defined homomorphisms

$$
\psi_{D}: \mathfrak{A}_{D} \rightarrow \mathfrak{A}_{D-n},
$$

which are characterised, at least on $\mathbf{U}_{D}$, by the conditions

- $\psi_{D}\left(E_{i}(D)\right)=E_{i}(D-n)$,

- $\psi_{D}\left(F_{i}(D)\right)=F_{i}(D-n)$,

- $\psi_{D}\left(K_{a}(D)\right)=v^{a \cdot b_{0}} K_{a}(D-n)$,

where $\boldsymbol{b}_{0}$ has all entries equal to 1 . It follows that if we work with the root datum $\left(X^{\prime}, Y^{\prime}\right)$, i.e., with $\widehat{\mathfrak{s l}}_{n}$, then the maps $\psi_{D}$ and $\phi_{D}: \dot{\mathbf{U}} \rightarrow \mathfrak{A}_{D, n, n}$ are compatible, that is $\psi_{D+n} \circ \phi_{D+n}=\phi_{D}$.

Let $\hat{\mathbf{U}}=\lim _{D} \mathfrak{A}_{D}$, where the limit is taken over the projective system given by the maps $\left(\psi_{D}\right)_{D \in \mathbb{N}}$ described above. Since the maps $\phi_{D}$ are compatible with this system, there is a unique map $\phi: \dot{\mathbf{U}} \rightarrow \hat{\mathbf{U}}$, which factors each of the maps $\phi_{D}$ through the canonical map $\hat{\mathbf{U}} \rightarrow \mathfrak{A}_{D}$. Theorem 5.2 allows us to give an alternative proof of the following injectivity result which is due to Lusztig [2000].

Proposition 7.1. The homomorphism $\phi$ is injective.

Proof. We first note that the inner product on $\dot{\mathbf{U}}$ is nondegenerate. While this is not explicitly stated in the book [Lusztig 1993], it follows easily from the results there. For example, one may use the results of $\$ 26.2$ and the nondegeneracy of the inner product defined in $\$ 19.1$ which is established in Lemma 19.1.4 (all references in this sentence are to sections of [Lusztig 1993]). Now suppose that $u$ is in the kernel of $\phi$. Then for every $D$ we have $\phi_{D}(u)=0$, and hence by Theorem 5.2 we see that $u$ is in the radical of the inner product on $\dot{\mathbf{U}}$, and hence it follows that $u=0$.

The modified quantum group $\dot{\mathbf{U}}$ is equipped with a canonical basis $\dot{\mathbf{B}}$ which generalises the canonical basis of $\mathbf{U}^{-}$. We now show that the compatibility of the inner products can be used to give an essentially self-contained construction of this basis. Let $\mathbf{A}=\mathbb{Q}(v) \cap \mathbb{Q} \llbracket v^{-1} \rrbracket$ and let $\mathbb{B}^{ \pm}$be defined by

$$
\mathbb{B}^{ \pm}=\left\{b \in \dot{\mathbf{U}}_{\mathscr{A}}:(b, b) \in 1+v^{-1} \mathbf{A}, \bar{b}=b\right\} .
$$


We show that this set is a signed basis of $\dot{\mathbf{U}}$. (We will also be able to choose a basis within this set.)

Remark 7.2. Lusztig [1993] showed that $\dot{\mathbf{B}}$ is "almost orthonormal" for the inner product, from which one can also deduce the (weaker) nondegeneracy statement used in the proof of Proposition 7.1. We prefer the argument given above since we wish to give a geometric construction of the canonical basis which does not presuppose its existence.

We begin by showing that $\mathbb{B}^{ \pm}$is closely related to the bases $\mathfrak{B}_{D}$ of the algebras $\mathfrak{A}_{D}$.

Proposition 7.3. Let $b \in \mathbb{B}^{ \pm}$. Then there exists $\lambda$ such that $b \in \dot{\mathbf{U}} 1_{\lambda}$. If $k$ is the residue of $\sum_{i=1}^{n} \lambda_{i} \bmod n$ then there is a $p_{0}>0$ such that for all $p>p_{0}$ we have $\phi_{k+p n}(b) \in \pm \mathfrak{B}_{D}$. Conversely, if $b \in \dot{\mathbf{U}} 1_{\lambda}$ has $\phi_{D}(b) \in \pm \mathfrak{B}_{k+p n}$ for all $p>p_{1}$ (some $p_{1} \in \mathbb{N}$ ) then $b \in \mathbb{B}^{ \pm}$.

Proof. Suppose that $b \in \mathbb{B}^{ \pm}$. Then since the inner product $\dot{\mathbf{U}}$ is obtained as a limit from the inner products on $\mathfrak{A}_{D}$, we see that for large $p$ we have (in the notation of Section 4)

$$
\sum_{l=0}^{n-1}\langle b, b\rangle_{l+p n}=1 \bmod v^{-1} \mathbb{Z}\left[v^{-1}\right] .
$$

Now for each $l,(0 \leq l \leq n-1)$ set $x_{l+p n}=\phi_{l+p n}(b)$. It is clear that $x_{l+p n}$ is bar-invariant (for the bar involution on $\mathfrak{A}_{l+p n}$, see Remark 6.2), and lies in $\mathfrak{A}_{l+p n, \mathfrak{A}}$. Thus we may write $x_{l+p n}=\sum_{i \in I} a_{i}\left\{A_{i}\right\}$ for some $a_{i} \in \mathscr{A}$ and $A_{i} \in \mathfrak{S}_{l+p n, n, n}^{\text {ap }}$, where $\bar{a}_{i}=a_{i}$. Now suppose that $a_{i} \in v^{m} \mathbb{Z}\left[v^{-1}\right]$ for all $i \in I$, and $m$ is minimal with this property. Then, using the "almost orthonormality" property that $\left(\left\{A_{i}\right\},\left\{A_{j}\right\}\right) \in$ $\delta_{i, j}+v^{-1} \mathbb{Z}\left[v^{-1}\right]$ (see Lemma 6.1), we see that if $J \subset I$ denotes the subset consisting of those $i$ with $a_{i}=c_{i} v^{m}+\cdots$, where $c_{i} \neq 0$, then

$$
\left(x_{l+p n}, x_{l+p n}\right)_{l+p n}=\left(\sum_{i \in J} c_{i}^{2}\right) v^{2 m}+\text { lower order terms. }
$$

In particular, since $\left(x_{l+p n}, x_{l+p n}\right) \in \mathbb{Z}\left[v^{-1}\right]$ we must have $m=0$. But then since $x_{l+p n}$ is bar-invariant, we must have $a_{i} \in \mathbb{Z}$ for each $i \in I$. Now since

$$
\sum_{k=1}^{n-1}\left(x_{k+p n}, x_{k+p n}\right) \in 1+v^{-1} \mathbb{Z}\left[v^{-1}\right],
$$

it follows that in fact there is a $k \in\{0,1, \ldots, n-1\}$ such that $x_{l+p n}=0$ for $l \neq k$ and $x_{k+p n}= \pm\{A\}$ for some $A \in \mathfrak{S}_{k+p n, n, n}$. Indeed the same argument shows that the signed basis $\pm \mathfrak{B}_{D}$ is characterised by the properties that its elements are 
bar-invariant, integral, and almost orthonormal. Note also that if $\lambda=c(A) \bmod \mathbb{Z} \boldsymbol{b}_{0}$ it is then easy to see that $b=b 1_{\lambda}$ as claimed in the statement of the lemma.

The converse is easier, since we know that $\dot{\mathbf{U}}$ injects into the inverse limit of the $\mathfrak{A}_{D}$, so that if $\phi_{D}(b) \in \mathfrak{A}_{D, \mathscr{A}}$ for all $D \equiv k \bmod n$, then $b \in \dot{\mathbf{U}}_{\mathscr{A}}$, and bar invariance and the condition on $(b, b)$ is also evident.

To extract a basis from $\mathbb{B}^{ \pm}$we need to recall some results of Lusztig. For this we need some definitions. Let $\mathfrak{S}_{D, n, n}^{-}$, be the set of all $B \in \mathfrak{S}_{D, n, n}$ such that $b_{i j}=0$ for $i>j$. Let $\mathfrak{S}_{D, n, n}^{+}$be the set of all $B \in \mathfrak{S}_{D, n, n}$ such that $b_{i j}=0$ for all $i<j$. Given $A \in \mathfrak{S}_{D, n, n}$ we may define $A^{+}$and $A^{-}$in $\mathfrak{S}_{D, n, n}^{+}$and $\mathfrak{S}_{D, n, n}^{-}$respectively by

$$
\begin{array}{lll}
a_{i j}^{-}=a_{i j} \text { if } i<j, & a_{i j}^{-}=0 \text { if } i>j, & a_{i i}^{-}=\sum_{j \in \mathbb{Z}, i \geq j} a_{i j}, \\
a_{i j}^{+}=a_{i j} \text { if } i>j, & a_{i j}^{+}=0 \text { if } i>j, & a_{i i}^{-}=\sum_{k \in \mathbb{Z}, k \leq i} a_{k i} .
\end{array}
$$

Lemma 7.4. Let $A \in \mathfrak{S}_{D, n, n}$.

(1) If $A \in \mathfrak{S}_{D, n, n}^{ \pm}$then $\psi_{D}(\{A\})=\{A-I\}$.

(2) For any $A \in \mathfrak{S}_{D, n, n}$ we have

$$
\left\{A^{-}\right\}\left\{A^{+}\right\}=\{A\}+\sum_{A^{\prime}<A} c_{A, A^{\prime}}\left\{A^{\prime}\right\},
$$

where $c_{A, A^{\prime}} \in \mathbb{Z}\left[v, v^{-1}\right]$.

(3) For any $A \in \mathfrak{S}_{D, n, n}$ we have

$$
\psi_{D}(\{A\})=\{A-I\}+\sum_{A^{\prime}<A} e_{A, A^{\prime}}\left\{A^{\prime}-I\right\},
$$

where $\{A-I\}$ is interpreted as 0 if $A-I$ does not lie in $\mathfrak{S}_{D, n, n}$, and likewise for $\left\{A^{\prime}-I\right\}$.

Proof. In [Lusztig 2000, §3.7] the elements of $A \in \mathfrak{S}_{D, n, n}^{ \pm}$are related to perverse sheaves on quiver varieties attached to the cyclic quiver, giving a geometric interpretation of part of the map from $\dot{\mathbf{U}}$ to $\mathfrak{A}_{D, n, n}$ (see also Section 6 and [Lusztig 1999, $\S 5]$ for more details). From this and the compatibility of the maps $\phi_{D}$ and $\psi_{D}$, part (1) readily follows. Part (2) is [Lusztig 1999, Proposition 4.11]. The last part follows by induction on the partial order $<$ using parts (1) and (2) together with the fact that $(A-I)^{ \pm}=A^{ \pm}-I$.

Definition 7.5. Next we note that the partial order $\leq$ has a combinatorial cousin $\preceq$ which we can make more explicit: Given $A, B \in \mathfrak{S}^{n, n}$ say $A \preceq B$ if for all $i<j \in \mathbb{Z}$ we have

$$
\sum_{r \leq i ; s \geq j} a_{r, s} \leq \sum_{r \leq i ; s \geq j} b_{r, s}
$$


and for any $i>j$ we have

$$
\sum_{r \geq i ; s \leq j} a_{r, s} \leq \sum_{r \geq i ; s \leq j} b_{r, s}
$$

It is easy to check that if $A, B \in \mathfrak{S}_{D, n, n}$ and $A \leq B$ then $A \preceq B$ (see for example [Beilinson et al. 1990, Lemma 3.6] and [Lusztig 1999, §1.6]). Also, if we write ${ }_{p} A=A+p I$, then it is clear that $A \preceq B$ if and only if ${ }_{p} A \preceq{ }_{p} B$. Moreover, crucially in what follows, given $A \in \mathfrak{S}^{n, n}$ the set

$$
\left\{B \in \mathfrak{S}^{n, n}: B \preceq A, r(B)=r(A), c(B)=c(A)\right\}
$$

is finite.

We now resolve the ambiguity of signs in the definition of $\mathbb{B}^{ \pm}$and extract a basis from the signed basis $\mathbb{B}^{ \pm}$.

\section{Corollary 7.6. Let}

$$
\mathbb{B}=\left\{b \in \mathbb{B}^{ \pm}: \phi_{D}(b) \in \mathfrak{B}_{D} \cup\{0\} \text { for all } D \gg 0\right\} .
$$

Then $\mathbb{B}^{ \pm}=\mathbb{B} \sqcup(-\mathbb{B})$. Moreover, if $\phi_{k+p n}(b)=\left\{A_{k+p n}\right\}$, where $A_{k+p n} \in \mathfrak{S}_{D, n, n}$ for all $p>p_{0}$ say, then $A_{k+(p+1) n}=A_{k+p n}+I$.

Proof. It is only necessary to show that $\mathbb{B}$ is well-defined. Suppose $b \in \mathbb{B}^{ \pm}$. The previous proposition shows that if $b=b 1_{\lambda}$, and $k=\sum_{i=1}^{n} \lambda_{i}$, then for large enough $p$, say $p \geq p_{0}$, we have $\phi_{k+p n}(b) \in \pm \mathfrak{B}_{k+p n}$, and moreover $\phi_{D}(b)=0$ if $D$ is not congruent to $k$ modulo $n$. Thus we have $\phi_{k+p n}(b)=\epsilon_{k+p n}\left\{A_{k+p n}\right\}$ where $\epsilon_{k+p n} \in\{ \pm 1\}$ and $A_{k+p n} \in \mathfrak{S}_{k+p n, n, n}$ for all $p \geq p_{0}$. But now by part (3) of Lemma 7.4 we have

$$
\psi_{k+(p+1) n}\left(\left\{A_{k+(p+1) n}\right\}\right)=\left\{A_{k+(p+1) n}-I\right\}+\sum_{B \preceq A_{k+p n}} e_{B}\{B\}, \quad\left(e_{B} \in \mathscr{A}\right),
$$

whereas $\psi_{k+(p+1) n}\left(\phi_{k+(p+1) n}(b)\right)=\phi_{k+p n}(b)=\epsilon_{k+p n}\left\{A_{k+p n}\right\}$. Comparing these two expressions we conclude that $\epsilon_{k+(p+1) n}=\epsilon_{k+p n}$ and $\left\{A_{k+(p+1) n}\right\}=\left\{A_{k+p n}\right\}+I$ as claimed.

Corollary 7.7. The set $\mathbb{B}$ is almost orthonormal, that is

$$
\left(b_{1}, b_{2}\right) \in \delta_{b_{1}, b_{2}}+v^{-1} \mathbb{Z} \llbracket v^{-1} \rrbracket .
$$

Thus the set $\mathbb{B}$ is linearly independent.

Proof. Let $b_{1}, b_{2} \in \mathbb{B}$. Take $\lambda \in X^{\prime}$ so that $b_{1}=b_{1} 1_{\lambda}$. Then either $b_{2} 1_{\lambda}=0$, in which case the corollary holds trivially, or $b_{2}=b_{2} 1_{\lambda}$. In that case, we see from 
Corollary 7.6 that we may find a $p_{0} \in \mathbb{N}$ and $A, B \in \mathfrak{S}^{n, n}$ so that $\phi_{p_{0}+p n}(b)=\left\{{ }_{p} A\right\}$ and $\phi_{p_{0}+p n}\left(b_{2}\right)=\left\{{ }_{p} B\right\}$ for all $p \geq 0$. But then it follows from Lemma 6.1 that

$$
\left(\left\{{ }_{p} A\right\},\left\{{ }_{p} B\right\}\right) \in \delta_{A, B}+v^{-1} \mathbb{Z} \llbracket v^{-1} \rrbracket .
$$

for all $p$, and hence taking the limit we obtain the same result for $b_{1}, b_{2}$. To see that this implies the linear independence of the set $\mathbb{B}$, consider a dependence involving the minimal number of elements of $\mathbb{B}$ :

$$
\sum_{j=1}^{k} p_{j} b_{j}=0
$$

where by clearing denominators if necessary we may assume that $p_{k} \in \mathbb{Z}\left[v, v^{-1}\right]$ (and by minimality they are all nonzero) and $b_{k} \in \mathbb{B}$. We may moreover assume, multiplying through by an appropriate power of $v$, that $p_{i}=n_{i}+v^{-1} \mathbb{Z}\left[v^{-1}\right]$, where $n_{i} \in \mathbb{Z}$, and, reordering if necessary, that $n_{1} \neq 0$. Pick $D$ large enough so that

$$
\sum_{l=0}^{n-1}\left(\phi_{D}\left(b_{r}\right), \phi_{D}\left(b_{s}\right)\right)_{D+l}=\left(b_{r}, b_{s}\right) \quad \bmod v^{-1} \mathbb{Z} \llbracket v^{-1} \rrbracket \quad \text { for } \quad 1 \leq r, s \leq k .
$$

and moreover that for each $j$ we have $\phi_{D}\left(b_{j}\right)=\left\{B_{j}\right\}$ for some $B_{j} \in \mathfrak{S}_{D, n, n}$. Then

$$
\begin{aligned}
0 & =\left(\sum_{r=1}^{k} p_{r} b_{r}, \sum_{s=1}^{k} p_{s} b_{s}\right)=\sum_{1 \leq r, s \leq k} p_{r} p_{s}\left(b_{r}, b_{s}\right) \\
& \equiv \sum_{1 \leq r, s \leq k} p_{r} p_{s}\left(\left\{B_{r}\right\},\left\{B_{s}\right\}\right)_{D} \bmod v^{-1} \mathbb{Z}\left[v^{-1}\right] \\
& \equiv \sum_{1 \leq r \leq k} n_{r}^{2} \bmod v^{-1} \mathbb{Z}\left[v^{-1}\right],
\end{aligned}
$$

which is a contradiction, since $n_{1} \neq 0$.

We now show that if $A \in \mathfrak{S}^{n, n}$ then for large enough $p$ there is a unique $b \in \mathbb{B}$ such that $\phi_{D}(b)=\left\{{ }_{p} A\right\}$ (where $D=\sum_{i \in[1, n], j \in \mathbb{Z}} a_{i j}+p n$ ), and hence by Corollary 7.6 it will also follows that for large enough $p$ we have $\psi_{D}(\{p A\})=\{p-1 A\}$. We need to recall the relation between the canonical basis $\mathbf{B}$ of $\mathbf{U}^{-}$and $\mathfrak{B}_{D}$. Recall from Section 6 that the representation theory of the cyclic quiver allows us to parametrise

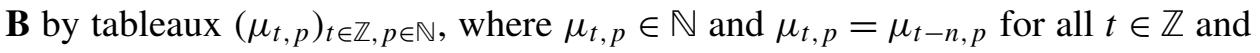
for fixed $t$ only finitely many of the $\mu_{t . p}$ are nonzero. The $v$-graded part $\mathbf{B}_{v}$ is then indexed by $\Sigma_{v}$.

The correspondence described in Section 6 gives a bijection between those $\left(\mu_{t, p}\right)$ in $\Sigma_{v}$ satisfying

$$
\mu_{i, 1}+\mu_{i, 2}+\cdots \leq a_{i} \text { for all } i
$$


and the orbits in corresponding to matrices $B \in \mathfrak{S}_{D, n, n}^{-}$with $r(B)=\boldsymbol{a}$. (Note that if the integers $a_{i}$ are sufficiently positive, this gives an injection from $\mathbf{B}_{v}$ into $\mathfrak{S}_{D, n, n}^{-}$.) Using the same correspondence, composed with the transpose map $\Psi$, we obtain a similar correspondence between (appropriate subsets of) $\mathbf{B}_{v}$ and elements of $\mathfrak{S}_{D, n, n}^{+}$. These can be combined to give a correspondence between the set of triples $\mathscr{T}=\left\{\left(b_{1}, b_{2}, \lambda\right): b_{1}, b_{2} \in \mathbf{B}, \lambda \in X\right\}$ and elements of $\mathfrak{S}^{n, n}$ as follows: the elements $b_{1}, b_{2}$ corresponds to tableau $\left(\mu_{t, p}\right)$ and $\left(\rho_{t, p}\right)$ say, and we define $A=A\left(b_{1}, b_{2}, \lambda\right) \in \mathscr{G}^{n, n}$ by setting

$$
a_{i j}= \begin{cases}\mu_{i, j-i} & \text { if } i<j \\ \rho_{j, i-j} & \text { if } i>j \\ \lambda_{i}-\sum_{t \geq 1} \mu_{i, t}-\sum_{s \geq 1} \rho_{i} & \text { if } i=j\end{cases}
$$

We will write $b_{A}$ for the element $b_{1}^{+} 1_{\lambda} b_{2}^{-} \in \dot{\mathbf{U}}$, and ${ }_{D} b_{A}$ for its image under $\phi_{D}$. It follows from the [Lusztig 1999, §5] and [Lusztig 1999, Proposition 4.11] that if $\sum_{i=1}^{n} \lambda_{i}=D$ and the entries of $A\left(b_{1}, b_{2}, \lambda\right)$ are all nonnegative, then

$$
{ }_{D} b_{A}=\left\{{ }_{p} A\right\}+\sum_{B \prec{ }_{p} A} e_{B,{ }_{p} A}\{B\}
$$

Proposition 7.8. Let $A \in \mathfrak{S}^{n, n}$. For large enough $p$ we have

$$
\psi_{D}(\{p A\})=\left\{{ }_{p-1} A\right\}
$$

Proof. Via the bijection described above between $\mathscr{T}$ and $\mathfrak{S}^{n, n}$, we may find a subset $\mathscr{T}_{\boldsymbol{a}}$ of $\mathscr{T}$ such that $\left\{A\left(b_{1}, b_{2}, \boldsymbol{a}\right)\right\}$ is a basis of $\mathfrak{A}_{D}\left[\boldsymbol{i}_{\boldsymbol{a}}\right]$ as $\left(b_{1}, b_{2}, \boldsymbol{a}\right)$ runs over the set $\mathscr{T}_{\boldsymbol{a}}$. Then the elements ${ }_{D} b_{A}$ are clearly also a basis of $\mathfrak{A}_{D}\left[\boldsymbol{i}_{\boldsymbol{a}}\right]$ since they are related to the elements $\{A\}$ by an upper triangular matrix, and moreover they satisfy

(1) $\overline{{ }_{D} b_{A}}={ }_{D} b_{A}$,

(2) ${ }_{D} b_{A} \in \mathbf{U}_{D, \mathscr{A}}$.

As in the proof of Proposition 7.3, the basis $\left\{\{A\}: A \in \mathfrak{S}_{D, n, n}\right\}$ is characterised up to sign by the properties of being bar-invariant, integral (that is, contained in $\mathbf{U}_{D, \mathscr{A}}$ ), and being almost orthonormal, so that

$$
(\{A\},\{B\})_{D} \in \delta_{A, B}+v^{-1} \mathbb{Z}\left[v^{-1}\right] .
$$

(In fact, Proposition 7.3 shows that less than this characterises $\pm \mathfrak{B}_{D}$ ).

We now show that one can obtain $\{A\}$ from ${ }_{D} b_{A}$ by a Gram-Schmidt style process. Indeed if $A$ is minimal for the ordering $\preceq$, then clearly $\{A\}=b_{A}$. Thus we consider the following claim: 
- For each $A \in \mathfrak{S}^{n, n}$, there is a $p_{0} \in \mathbb{Z}$ such that for all $p>p_{0}$ we have

$$
\left\{{ }_{p} A\right\}={ }_{D} b_{A}+\sum_{A^{\prime}<A} d_{A^{\prime}, A D} b_{A^{\prime}} .
$$

where $d_{A^{\prime}, A} \in \mathscr{A}$ do not depend on $p$, and $D=p n+\sum_{i, j: 1 \leq i \leq n} a_{i j}$.

The proof of the proposition now follows immediately since $\psi_{D}\left({ }_{D} b_{A}\right)={ }_{D-n} b_{A}$. We show this by induction on $\preceq$ : if $A$ is minimal, then (7-1) implies that $b_{A}=\{A\}$, and we are done. Thus suppose that the result is known for all $B \prec A$, and let $I$ be the (finite) set

$$
\left\{B \in \mathfrak{S}^{n, n}: B \preceq A, r(A)=r(B), c(A)=c(B)\right\} .
$$

Now for $x$ in the span of $\{\{B\}: B \in I\}$, set $N(x)=\max \left\{v(x,\{B\})_{D}: B \in I, B \neq A\right\}$, where for $f \in \mathscr{A}$ we let $v(f)$ denote the highest power of $v$ occurring in $f$. Let $N=N\left(b_{A}\right)$, and suppose that $N \geq 0$. Let $J$ denotes the subset of $I$ for which $v\left(b_{A},\{B\}\right)=N$, so that if $B \in J$ we have

$$
\left(b_{A},\{B\}\right)_{D}=c_{B} v^{N}+\text { lower order terms } \quad\left(c_{B} \in \mathbb{Z}\right) .
$$

Now $(\{B\},\{B\})_{D} \in 1+v^{-1} \mathbb{Z}\left[v^{-1}\right]$, so we may recursively solve for

$$
a_{B} \in v^{-N} \mathbb{Z}[v] \cap \mathbb{Z}\left[v^{-1}\right]
$$

such that $a_{B} \cdot(\{B\},\{B\})_{D} \in 1+v^{-N-1} \mathbb{Z}\left[v^{-1}\right]$. It follows immediately that we may find $e_{B} \in \mathscr{A}$ such that $\bar{e}_{B}=e_{B}$ and $e_{B}=c_{B} v^{N} a_{B} \bmod v^{-1} \mathbb{Z}\left[v^{-1}\right]$. Then we set

$$
b_{A}^{\prime}=b_{A}-\sum_{B \in J} e_{B}\{B\} .
$$

It follows from the almost orthonormality of the $\{B\}$ that $\left(b_{A}^{\prime},\{B\}\right)_{D} \in v^{N-1} \mathbb{Z}\left[v^{-1}\right]$, and $b_{A}^{\prime}$ is again bar-invariant, lies in $\mathbf{U}_{D, \mathscr{A}}$, and satisfies $N\left(b_{A}^{\prime}\right)<N$. We may thus iterate this construction to obtain an element $b_{A}^{\prime \prime}$ which has $N\left(b_{A}^{\prime \prime}\right) \leq-1$, is bar-invariant, and lies in $\mathbf{U}_{D, \mathscr{A}}$. But then we claim that we must have $b_{A}^{\prime \prime}=\{A\}$. Indeed we know from (7-1) that we can write

$$
b_{A}^{\prime \prime}=\{A\}+\sum_{B \prec A} f_{B}\{B\}
$$

for some $f_{B} \in \mathscr{A}$ with $\bar{f}_{B}=f_{B}$. If it is not the case that $f_{B}=0$ for all $B$, then there is some $B$ with $v\left(f_{B}\right) \geq 0$ maximal, whence we see that $v\left(b_{A}^{\prime \prime},\{B\}\right)_{D} \geq 0$ which is a contradiction. Thus $b_{A}^{\prime \prime}=\{A\}$ as required.

Now examining the above process, we see that it uses only the values of $\left(b_{A},\{B\}\right)_{D}$ down to order to $v^{-N\left(b_{A}\right)} \mathbb{Z}\left[v^{-1}\right]$, and by induction we see that these, for large enough $p$ are determined by the values of $\left(b_{A}, b_{B}\right)_{D}$, down to some possibly lower order (determined by the coefficients $\left.d_{B, C}\right)$. Since the values of $\left(b_{A}, b_{B}\right)_{D}$ converge in $\mathbb{Z}\left(\left(v^{-1}\right)\right)$ we see that we may find a large enough $p_{0}$ so that $\left\{_{p} A\right\}$ is 
a linear combination of $\left\{\phi_{D}\left(b_{A^{\prime}}\right): A^{\prime} \preceq A\right\}$ with coefficients independent of $p$ as required.

We can now show that the set $\mathbb{B}$ is a basis of $\dot{\mathbf{U}}$.

Theorem 7.9. $\mathbb{B}$ is a basis of $\dot{\mathbf{U}}$.

Proof. Notice first that given any $b \in \mathbb{B}$, Proposition 7.3 implies that $\phi_{D}(b) \in$ $\mathfrak{B}_{D} \cup\{0\}$ for large enough $D$, and is nonzero provided $D$ has a fixed residue modulo $n$. By Corollary 7.7 we know that the elements of $\mathbb{B}$ are linearly independent, so we need only show that they span $\dot{\mathbf{U}}$. To do this it is enough to show that the element $b_{1}^{+} b_{2}^{-} 1_{\lambda}$ for $b_{1}, b_{2} \in \mathbf{B}$ and $\lambda \in X$ lie in the span of $\mathbb{B}$, since they form a basis for $\dot{\mathbf{U}}$. But the claim in the proof of Proposition 7.8 shows that we may find an element of $\mathbb{B}$ which is a linear combination of such basis elements with leading coefficient 1 , so that the matrix relating the two sets is invertible and $\mathbb{B}$ indeed spans $\dot{\mathbf{U}}$.

Remark 7.10. The results of [Lusztig 1993, §26.3] then show that $\mathbb{B}=\dot{\mathbf{B}}$, and thus the results of this section give a new proof of the conjecture made in [Lusztig 1999, $\S 9.3$, which was originally proved by Schiffmann and Vasserot [2000]. Our goal here was to construct the canonical basis purely within the context of the inverse system $\mathbf{U}_{D}$; thus, unlike Schiffmann and Vasserot, we do not need to assume the existence of $\dot{\mathbf{B}}$, nor use any properties of crystal bases. It should be noted however that by using results of Kashiwara on global crystal bases, those authors have obtained a more precise result (also conjectured in [2000]) saying that the maps $\phi_{D}$ are all compatible with the canonical basis; i.e., if $b \in \dot{\mathbf{B}}$ then $\phi_{D}(b) \in \mathfrak{B}_{D} \cup\{0\}$, and moreover the kernel of $\phi_{D}$ is spanned by a subset of $\dot{\mathbf{B}}$. The results of this section show that this theorem would also follow if we could show that the maps $\psi_{D}$ are compatible with the bases $\mathbf{B}_{D}$ and $\mathbf{B}_{D-n}$, a question which can be phrased purely geometrically (in terms of perverse sheaves). Note that it is not true that the maps $\psi_{D}$ send $\mathfrak{B}_{D}$ to $\mathfrak{B}_{D-n} \cup\{0\}$, as was pointed out already in [Lusztig 2000, 1.12]. It is possible to give a construction of the maps $\psi_{D}$ in the context of perverse sheaves on the ind-varieties $\mathscr{F}_{\boldsymbol{a}}$, (i.e., to show that there exists a functor on the derived category that preserves perverse sheaves (up to shift) and induces $\psi_{D}$ on the Grothendieck group which moreover is compatible with the "convolution" on $\left.\mathscr{K}_{D, n, n}\right)$, but it is not immediately clear why this functor preserves simple objects.

\section{A positivity result}

We may combine Theorem 5.2 and Proposition 6.5 to prove a positivity result for the inner product of two elements of $\dot{\mathbf{B}}$. This has been conjectured by Lusztig for all types.

Theorem 8.1. Let $b_{1}, b_{2} \in \dot{\mathbf{B}}$. Then

$$
\left(b_{1}, b_{2}\right) \in \mathbb{N} \llbracket v^{-1} \rrbracket \cap \mathbb{Q}(v) .
$$


Proof. We may assume that there is a $\lambda \in X$ such that $b_{1} 1_{\lambda}=b_{1}$, and $b_{2} 1_{\lambda}=b_{2}$. Let $k \in\{0,1, \ldots, n-1\}$ be such that $\sum_{j=1}^{n} \lambda_{j}=k \bmod n$. Then

$$
\left(b_{1}, b_{2}\right)=\lim _{p \rightarrow \infty}\left(\phi_{k+p n}\left(b_{1}\right), \phi_{k+p n}\left(b_{2}\right)\right)_{k+p n}
$$

By Proposition 7.3 we know that for all large enough $D$ we have $\phi_{D}\left(b_{1}\right), \phi_{D}\left(b_{2}\right)$ are in $\mathfrak{B}_{D}$, hence it is clear from (6-1) that

$$
\left(\phi_{k+p n}\left(b_{1}\right), \phi_{k+p n}\left(b_{2}\right)\right)_{k+p n} \in \mathbb{N}\left[v, v^{-1}\right] .
$$

However, it follows also from Lemma 6.1 that the left-hand side is in fact in $\mathbb{N}\left[v^{-1}\right]$ (this can also be seen directly, using the definition of intersection cohomology sheaves). Hence $\left(b_{1}, b_{2}\right)$ is the limit of elements of $\mathbb{N}\left[v^{-1}\right]$, and the statement follows.

Remark 8.2. All the results of this paper have analogues for the nonaffine case, which can be proved in exactly the same way. The module $V$ is replaced by a $D$-dimensional vector space over $\mathbb{k}$, and the space $\mathscr{F}^{n}$ of $n$-step periodic lattices should be replaced by the space of n-step flags in that vector space. In this case the algebra corresponding to $\mathbf{U}_{D}$ is actually equal to the algebra analogous to $\mathfrak{A}_{D}$, hence the results are sometimes more straightforward.

\section{Acknowledgements}

This paper is based on a chapter of my thesis, written under the direction of George Lusztig. I would like to thank him both for posing the problem that led to this paper and for our many conversations about the contents of this paper and much else besides. I would also like to thank Jared Tanner for a useful conversation.

\section{References}

[Beilinson et al. 1990] A. A. Beilinson, G. Lusztig, and R. MacPherson, "A geometric setting for the quantum deformation of GL", Duke Math. J. 61:2 (1990), 655-677. MR 1074310 (91m:17012) Zbl 0713.17012

[Bernstein and Lunts 1994] J. Bernstein and V. Lunts, Equivariant sheaves and functors, Lecture Notes in Mathematics 1578, Springer, Berlin, 1994. MR 95k:55012 Zbl 0808.14038

[Ginzburg and Vasserot 1993] V. Ginzburg and É. Vasserot, "Langlands reciprocity for affine quantum groups of type $A_{n}$ ", Internat. Math. Res. Notices 1993:3 (1993), 67-85. MR 1208827 (94j:17011 Zbl 0785.17014

[Grojnowski and Lusztig 1993] I. Grojnowski and G. Lusztig, "A comparison of bases of quantized enveloping algebras", pp. 11-19 in Linear algebraic groups and their representations (Los Angeles, CA, 1992), edited by R. S. Elman et al., Contemp. Math. 153, Amer. Math. Soc., Providence, RI, 1993. MR 1247495 (94m:17012) Zbl 1009.17502

[Kashiwara 1991] M. Kashiwara, "On crystal bases of the $Q$-analogue of universal enveloping algebras”, Duke Math. J. 63:2 (1991), 465-516. MR 93b:17045 Zbl 0739.17005 
[Kashiwara and Schapira 1994] M. Kashiwara and P. Schapira, Sheaves on manifolds, Grundlehren Math. Wiss. 292, Springer, Berlin, 1994. MR 95g:58222

[Lang and Weil 1954] S. Lang and A. Weil, "Number of points of varieties in finite fields", Amer. J. Math. 76 (1954), 819-827. MR 16,398d Zbl 0058.27202

[Lusztig 1990] G. Lusztig, "Canonical bases arising from quantized enveloping algebras", J. Amer. Math. Soc. 3:2 (1990), 447-498. MR 90m:17023 Zbl 0703.17008

[Lusztig 1991] G. Lusztig, "Quivers, perverse sheaves, and quantized enveloping algebras", J. Amer. Math. Soc. 4:2 (1991), 365-421. MR 1088333 (91m:17018) Zbl 0738.17011

[Lusztig 1992] G. Lusztig, "Canonical bases in tensor products", Proc. Nat. Acad. Sci. U.S.A. 89:17 (1992), 8177-8179. MR 93j:17033 Zbl 0760.17011

[Lusztig 1993] G. Lusztig, Introduction to quantum groups, vol. 110, Progress in Mathematics, Birkhäuser Boston Inc., Boston, MA, 1993. MR 1227098 (94m:17016)

[Lusztig 1999] G. Lusztig, "Aperiodicity in quantum affine $\mathfrak{g l}_{n}$ ", Asian J. Math. 3:1 (1999), 147-177. MR 1701926 (2000i:17027)

[Lusztig 2000] G. Lusztig, "Transfer maps for quantum affine $\mathfrak{s l}_{n}$ ", pp. 341-356 in Representations and quantizations (Shanghai, 1998), edited by Z. L. Jian-pan Wang, China High. Educ. Press, Beijing, 2000. MR 1802182 (2002f:17026)

[Schiffmann and Vasserot 2000] O. Schiffmann and E. Vasserot, "Geometric construction of the global base of the quantum modified algebra of $\widehat{\mathfrak{g l}}_{n}$ ", Transform. Groups 5:4 (2000), 351-360. MR 1800532 (2001k:17029) Zbl 0978.17007

Communicated by Edward Frenkel

Received 2010-10-03 Revised 2011-05-08 Accepted 2011-06-30

mcgerty@maths.ox.ac.uk

Mathematical Institute, University of Oxford, 24-29 St Giles', Oxford, OX13LB, United Kingdom 


\section{Algebra \& Number Theory}

msp.berkeley.edu/ant

\section{EDITORS}

MANAGING EDITOR

Bjorn Poonen

Massachusetts Institute of Technology

Cambridge, USA

\author{
EDITORIAL BOARD CHAIR \\ David Eisenbud \\ University of California \\ Berkeley, USA
}

\section{BOARD OF EDITORS}

Georgia Benkart

Dave Benson

Richard E. Borcherds

John H. Coates

J-L. Colliot-Thélène

Brian D. Conrad

Hélène Esnault

Hubert Flenner

Edward Frenkel

Andrew Granville

Joseph Gubeladze

Ehud Hrushovski

Craig Huneke

Mikhail Kapranov

Yujiro Kawamata

János Kollár

Yuri Manin

Barry Mazur

Philippe Michel
University of Wisconsin, Madison, USA

University of Aberdeen, Scotland

University of California, Berkeley, USA

University of Cambridge, UK

CNRS, Université Paris-Sud, France

University of Michigan, USA

Universität Duisburg-Essen, Germany

Ruhr-Universität, Germany

University of California, Berkeley, USA

Université de Montréal, Canada

San Francisco State University, USA

Hebrew University, Israel

University of Virginia, USA

Yale University, USA

University of Tokyo, Japan

Princeton University, USA

Northwestern University, USA

Harvard University, USA

École Polytechnique Fédérale de Lausanne
Susan Montgomery

Shigefumi Mori

Raman Parimala

Jonathan Pila

Victor Reiner

Karl Rubin

Peter Sarnak

Joseph H. Silverman

Michael Singer

Vasudevan Srinivas

J. Toby Stafford

Bernd Sturmfels

Richard Taylor

Ravi Vakil

Michel van den Bergh

Marie-France Vignéras

Kei-Ichi Watanabe

Andrei Zelevinsky

Efim Zelmanov
University of Southern California, USA

RIMS, Kyoto University, Japan

Emory University, USA

University of Oxford, UK

University of Minnesota, USA

University of California, Irvine, USA

Princeton University, USA

Brown University, USA

North Carolina State University, USA

Tata Inst. of Fund. Research, India

University of Michigan, USA

University of California, Berkeley, USA

Harvard University, USA

Stanford University, USA

Hasselt University, Belgium

Université Paris VII, France

Nihon University, Japan

Northeastern University, USA

University of California, San Diego, USA

\section{PRODUCTION}

contact@msp.org

Silvio Levy, Scientific Editor

See inside back cover or www.jant.org for submission instructions.

The subscription price for 2012 is US \$175/year for the electronic version, and \$275/year (+ \$40 shipping outside the US) for print and electronic. Subscriptions, requests for back issues from the last three years and changes of subscribers address should be sent to Mathematical Sciences Publishers, Department of Mathematics, University of California, Berkeley, CA 94720-3840, USA.

Algebra \& Number Theory (ISSN 1937-0652) at Mathematical Sciences Publishers, Department of Mathematics, University of California, Berkeley, CA 94720-3840 is published continuously online. Periodical rate postage paid at Berkeley, CA 94704, and additional mailing offices.

ANT peer review and production are managed by EditFLOW ${ }^{\circledR}$ from Mathematical Sciences Publishers.

PUBLISHED BY

mathematical sciences publishers

http://msp.org/

A NON-PROFIT CORPORATION

Typeset in IATEX

Copyright @2012 by Mathematical Sciences Publishers 


\section{Algebra \& Number Theory}

Volume $6 \quad$ No. $6 \quad 2012$

The smallest prime that does not split completely in a number field

XIANNAN LI

On the geometric realization of the inner product and canonical basis for quantum affine

1097 $\mathfrak{s l}_{n}$

\section{KEVIN MCGERTY}

Combinatorics of the tropical Torelli map

MELODY CHAN

Sonia Natale and Julia Yael PlavniK

Cusp form motives and admissible $G$-covers

DAN PETERSEN

Ideals of degree one contribute most of the height

AARON LEVIN and DAVID MCKINNON

Torsion des modules de Drinfeld de rang 2 et formes modulaires de Drinfeld 